\title{
Structural Geometry, Vibrational and Electronic Spectra Investigation on Naringin Molecule Using Experimental and Density functional theory calculation
}

\author{
Suresh $\mathbf{R}^{1}$, Balakumar $\mathbf{R}^{1}$, Krishnakumar $\mathbf{N}^{2}$, Saleem $\mathbf{H}^{2 *}$ and Subashchandrabose $\mathbf{S}^{1,3^{*}}$ \\ ${ }^{1}$ Centre for Research and Development, PRIST University, Thanjavur, Tamilnadu, India-613403 \\ ${ }^{2}$ Department of Physics, Annamalai University, Annamalai nagar, Tamil Nadu, India-608 002. \\ ${ }^{3}$ Centre for Functionalized Magnetic materials (FunMagMa), Immanuel Kant Baltic Federal University-236000, \\ Kaliningrad, Russia.
}

\begin{abstract}
Received: February 8, 2018; Accepted: February 24, 2018; Published: March 1, 2018
*Corresponding author: S. Subashchandrabose, Centre for Research and Development, PRIST University, Thanjavur, Tamilnadu, India-613403. Tel: +91 9976853476; E-mail: sscbphysics@gmail.com
\end{abstract}

\begin{abstract}
In the present investigation we investigate the structural, spectral and Molecular Orbitals (MO's) properties of Naringin compound. It is a bioflavonoid, present in the fruits; it is responsible for bitter taste in fruits. The Naringin has a flavanone-7-0-glycoside between the flavanone naringenin and the disaccharide neohesperidose. It consists of naringenin (ring A-C), D-glucose (ring D) and L-rhamnose (ring E). Our aim is to predict the molecular structure and investigate the insights of the Naringin molecule. Besides that to study the vibrational behavior of Naringin, the FT-IR and FT-Raman spectra were recorded in the ranges of $4000-400 \mathrm{~cm}^{-1}$ and $3500-50 \mathrm{~cm}^{-1}$ respectively, and to study the frontier molecular orbitals, the UVVisible spectrum was recorded in the range of $500-200 \mathrm{~nm}$. In addition to that the geometry of Naringin molecular structure was optimized by Density Functional Theory (DFT/B3LYP) calculation using 6-31g $(\mathrm{d}, \mathrm{p})$ level of basis set. For the optimized structure, vibrational spectral calculation was performed by the same level of theory calculation, furthermore, interpreted the calculated spectra of Naringin in terms of TED analysis. Moreover, to explain the inter and intra molecular charge transfer within the molecule the Natural Bond Orbital analysis (NBO) was performed, NBO analysis gave us a clear picture about hyperconjugative interaction energy between the donor (i) and acceptor(j) bond orbitals. In addition to that, the first order hyperpolarizability $(\beta 0)$, polarizability $(\alpha)$ and dipole moment $(\mu)$ of Naringin was computed. The frontier MO's (HOMO-LUMO) of Naringin was analyzed by TD-B3LYP/6-31g (d, p) level of basis set. Finally, the experimentally recorded results were compared with computed values, the agreement and discrepancies were studied carefully.
\end{abstract}

Keywords: FT-IR; FT-Raman; TED; NBO; Naringin;

\section{Introduction}

Flavonoids are naturally occurring phenolic compounds with a diverse range of bioactivities [1]. Among flavonoids, Naringin has great potential especially in the food and pharmaceutical industries due to their recognized antioxidant, anti-inflammatory, anti-ulcer, and hypocholesterolemic effects, whereas the
Naringenin has also shown anti-mutagenic and neuroprotective activities [2]. Naringin is a 'flavanone glycoside found in grape and citrus fruits. It has the distinct bitter taste of grapefruit juice. Its molecular formula is $\mathrm{C} 27 \mathrm{H} 32014$ and molecular weight is about $580.4 \mathrm{~g} / \mathrm{mol}$, it consists of L-rhamnose and D-glucose with Naringenin at the 7-carbon portion [3]. It is moderately soluble in water [4]. It has been reported to protect against oxygen free radical-stimulated $\mathrm{K}_{-}$permeability [5], metal chelating, antioxidant and free radical scavenging properties [6], Naringin, a major bioflavonoid in grapefruit, it has been shown to reduce radiation-induced damage to DNA [7]. It also acts as the inhibitor of VEGF (Vascular Endothelial Growth Factor) release, which causes angiogenesis and has been proven to be effective against ethanol injury in rats $[8,9]$.

Since the Naringin flavonoid having a wide application in above-mentioned fields, we investigate the structure geometry, vibrational spectra, Frontier Molecular Orbitals (FMOs), inter and intramolecular interactions by (experimental and computation) methods such as FT-IR, FT-Raman, UV-Visible spectra, DFT and Time dependent-DFT calculations. To the best of our knowledge, there is no such an article being published till now.

\section{Experimental Details}

\section{FT-Raman and FT-IR Spectra}

The solid form of Naringin compound was purchased from sigma Aldrich Company USA. The FT-Raman spectra was recorded in the region $3500-50 \mathrm{~cm}^{-1}$ using the FT-Raman spectrometer with Nd:YAG laser source, and $1064 \mathrm{~nm}$ as a excitation wavelength with spectral resolution of $4 \mathrm{~cm}^{-1}$ on a Bruker model IFS66V spectrophotometer equipped with an FRA 106 FT-Raman module accessory. The spectral measurements were carried out at Sree Chitra Tirunal Institute for Medical Sciences and Technology, Poojappura, Thiruvananthapuram, Kerala, India. The FT-IR spectrum of this compound was recorded in the region $400-4000 \mathrm{~cm}^{-1}$ on an IFS 66V spectrophotometer 
using the $\mathrm{KBr}$ pellet technique. The spectrum was recorded at room temperature, with a scanning speed of $10 \mathrm{~cm}^{-1}$ per minute and at the spectral resolution of $2.0 \mathrm{~cm}^{-1}$ in CSIL Laboratory, Annamalai University, and Tamilnadu, India. The ultraviolet absorption spectrum of Naringin was recorded in the range of 200-500 nm using a shimadzu UV-2401PC, UV-Visible recording spectrometer. The UV pattern is taken from a $10^{-5}$ molar solution of Naringin dissolved in methanol.

\section{Computational Details}

The entire calculations were performed at DFT level on a Pentium 1V/3.02 GHz personal computer using Gaussian 03W Program package [10], invoking gradient geometry optimization [11]. In this study, the DFT/B3LYP/6-31G (d, p) method has been utilized for the computation of molecular structure, vibrational frequencies and energies of optimized structures. By combining the results of the GAUSSVIEW program with symmetry considerations, vibrational frequency assignments were made with a high degree of accuracy and also the scaled quantum mechanics calculation was used to identify the vibrations [12, 13].

It should be noted that Gaussian 03W package able to calculate the Raman activity. The Raman activities were transformed into Raman intensities using Raint program by the expression [14]:

$$
\mathrm{I}_{\mathrm{i}}=10^{-12} \times\left(\mathrm{v}_{0}-\mathrm{V}_{\mathrm{i}}\right)^{4} \times \frac{1}{\mathrm{v}_{\mathrm{i}}} \times \mathrm{RA}
$$

Where Ii is the Raman intensity, $\mathrm{RA}_{\mathrm{i}}$ is the Raman scattering activities, $v i$ is the wavenumber of the normal modes and $v 0$ denotes the wavenumber of the excitation laser [15].

\section{Results and Discussion}

\section{Molecular Geometry}

Molecular structure of the Naringin was optimized by using DFT/B3LYP level of theory calculation using 6-31G (d, p) basis set. The optimized molecular structure along with numbering scheme is given in Figure 1 . It is belongs to $C_{1}$ point group symmetry. To the best of our literature survey, the crystal data of this molecule is not available till date [16]. So that, the optimized geometric parameters of Naringin is compared with Naringenin, quercetin and some flavones. It consists of L-rhamnose and D-glucose with naringenin at the 7-carbon, Naringin structure has 8 hydroxyl groups; it leads the molecule more inter-molecular hydrogen bonding interactions. The carbonyl present in naringenin forms intra-molecular hydrogen bonding interaction between carbonyl and hydroxyl groups. Due to this, the bond lengths of $\mathrm{C}_{3}-\mathrm{C}_{8}(1.424)$, $\mathrm{C}_{8}-\mathrm{C}_{10}(1.398)$ in ring $\mathrm{A}$ and $\mathrm{C}_{4}-\mathrm{C}_{5}(1.516 \AA)$ in ring $\mathrm{B}$ are positively elongated with $\mathrm{C}$-C bond lengths $(\sim 1.4 \AA)$ in ring $\mathrm{C}$ as well as with literature values [16].

Zhang et al. observed the $\mathrm{C}=\mathrm{O}$ and $\mathrm{C}-\mathrm{O}$ bond lengths are about 1.235 and $1.427 \AA$, respectively for 5, 6, 7, 4'-tetramethoxy isoflavone [17]. These observed values are in line with the calculated values $\left(\mathrm{C}_{4}=\mathrm{O}_{14}: 1.243\right.$ and $\left.\mathrm{C}_{8}-\mathrm{O}_{15}: 1.337 \AA\right)$ of Naringin molecule. Moreover, there is a shrink in bond length of $\mathrm{C}_{8}-\mathrm{O}_{15}$ due to delocalization of $\pi$-electron from $\mathrm{C}_{4}=\mathrm{O}_{14}$. On comparing the $\mathrm{C}-\mathrm{C}$ bond length in ring D, E with ring $\mathrm{A}, \mathrm{B}$ and $\mathrm{C}$ are higher $(\sim 1.5 \AA)$ due to the boat form of ring $\mathrm{D}$ and ring $\mathrm{E}$. In ring $\mathrm{A}$, the angle of $\mathrm{C}_{8}$ $\mathrm{O}_{15}-\mathrm{H}_{16}$ and $\mathrm{C}_{3}-\mathrm{C}_{8}-\mathrm{C}_{10}$ are about $106.52^{\circ}$ and $120.74^{\circ}$ respectively. These angles are negatively deviated from the angles of $\mathrm{C}_{24}$ $\mathrm{O}_{27^{-}}-\mathrm{H}_{28}\left(109.21^{\circ}\right)$ and $\mathrm{C}_{22}-\mathrm{C}_{24}-\mathrm{C}_{20}\left(119.78^{\circ}\right)$ is due to the electron localized in ring E. The calculated bond lengths, bond angles and dihedral angles are listed in Table S1 (Supporting information).

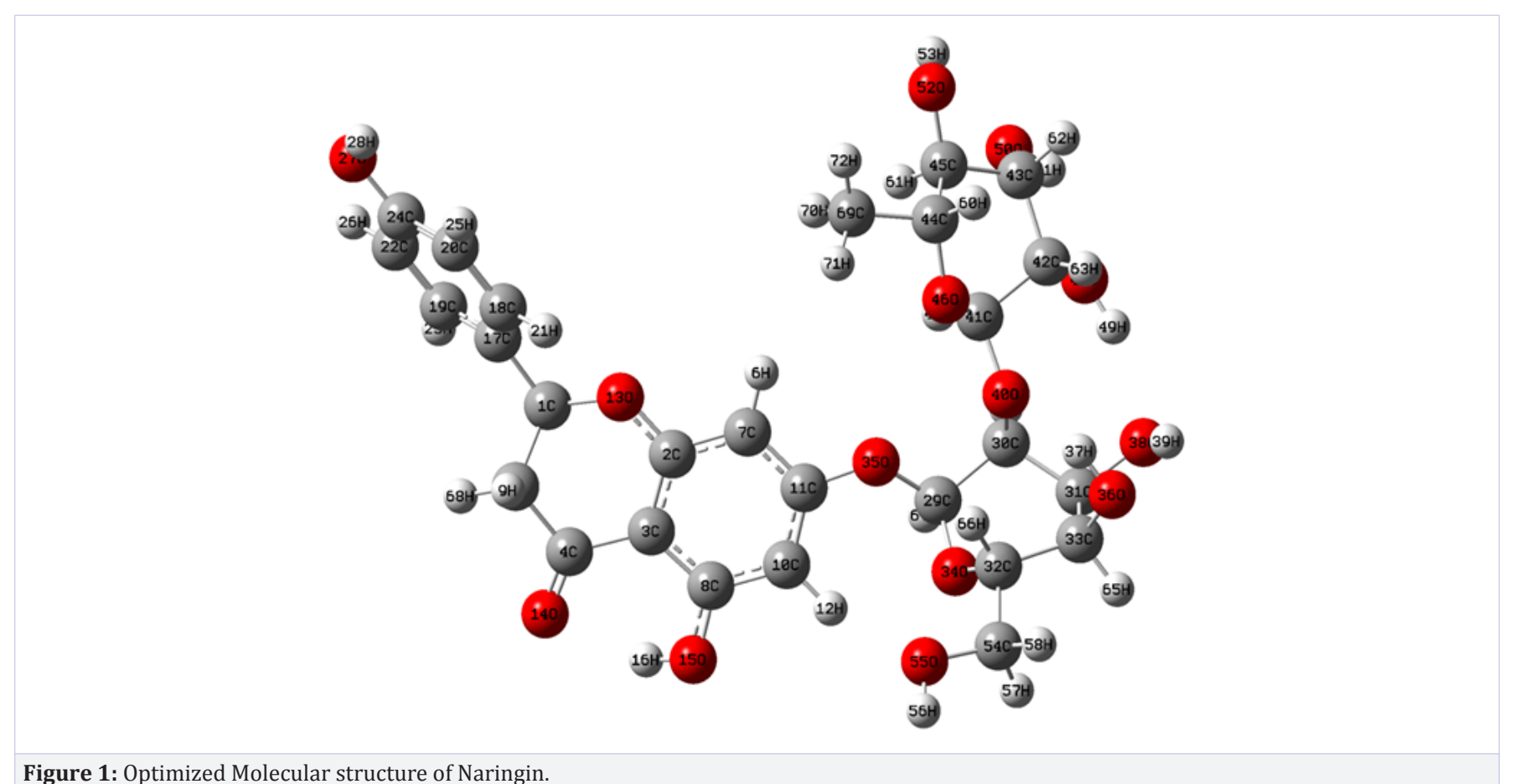

Figure 1: Optimized Molecular structure of Naringin. 
Table S1: The optimized bond lengths, bond angles and dihedral angles of Naringin using B3LYP/6-31G(d,p) basis set.

\begin{tabular}{|c|c|c|c|c|c|}
\hline Parameters & B3LYP/6-31G(d,p) & Exp. & Parameters & B3LYP/6-31G(d,p) & Exp. \\
\hline Bond lengths $(\AA ̊)$ & & & Bond length Contd... & & \\
\hline $\mathrm{C}_{1}-\mathrm{C}_{5}$ & 1.533 & & $\mathrm{C}_{30}-\mathrm{O}_{40}$ & 1.427 & \\
\hline $\mathrm{C}_{1}-\mathrm{O}_{13}$ & 1.447 & 1.376 & $\mathrm{C}_{30}-\mathrm{H}_{59}$ & 1.094 & \\
\hline $\mathrm{C}_{1}-\mathrm{C}_{17}$ & 1.509 & 1.467 & $\mathrm{C}_{31}-\mathrm{C}_{33}$ & 1.555 & \\
\hline $\mathrm{C}_{1}-\mathrm{H}_{73}$ & 1.100 & & $\mathrm{C}_{31}-\mathrm{O}_{38}$ & 1.424 & \\
\hline $\mathrm{C}_{2}-\mathrm{C}_{3}$ & 1.420 & 1.386 & $\mathrm{C}_{31}-\mathrm{H}_{64}$ & 1.095 & \\
\hline $\mathrm{C}_{2}-\mathrm{C}_{7}$ & 1.385 & 1.388 & $\mathrm{C}_{32}-\mathrm{C}_{33}$ & 1.544 & \\
\hline $\mathrm{C}_{2}-\mathrm{O}_{13}$ & 1.361 & 1.365 & $\mathrm{C}_{32}-\mathrm{O}_{34}$ & 1.442 & \\
\hline $\mathrm{C}_{3}-\mathrm{C}_{4}$ & 1.447 & 1.449 & $\mathrm{C}_{32}-\mathrm{C}_{54}$ & 1.521 & \\
\hline $\mathrm{C}_{3}-\mathrm{C}_{8}$ & 1.424 & 1.406 & $\mathrm{C}_{32}-\mathrm{H}_{66}$ & 1.097 & \\
\hline $\mathrm{C}_{4}-\mathrm{C}_{5}$ & 1.516 & 1.455 & $\mathrm{C}_{33}-\mathrm{O}_{36}$ & 1.428 & \\
\hline $\mathrm{C}_{4}-\mathrm{O}_{14}$ & 1.243 & 1.238 & $\mathrm{C}_{33}-\mathrm{H}_{65}$ & 1.095 & \\
\hline $\mathrm{C}_{5}-\mathrm{H}_{9}$ & 1.098 & & $\mathrm{O}_{36}-\mathrm{H}_{37}$ & 0.974 & \\
\hline $\mathrm{C}_{5}-\mathrm{H}_{68}$ & 1.093 & & $\mathrm{O}_{38}-\mathrm{H}_{39}$ & 0.977 & \\
\hline $\mathrm{H}_{6}-\mathrm{C}_{7}$ & 1.083 & 0.930 & $\mathrm{O}_{40}-\mathrm{C}_{41}$ & 1.443 & \\
\hline $\mathrm{C}_{7}-\mathrm{C}_{11}$ & 1.404 & 1.378 & $\mathrm{C}_{41}-\mathrm{C}_{42}$ & 1.536 & \\
\hline $\mathrm{C}_{8}-\mathrm{C}_{10}$ & 1.398 & 1.364 & $\mathrm{C}_{41}-\mathrm{O}_{46}$ & 1.394 & \\
\hline $\mathrm{C}_{8}-\mathrm{O}_{15}$ & 1.337 & 1.355 & $\mathrm{C}_{41}-\mathrm{H}_{47}$ & 1.095 & \\
\hline $\mathrm{C}_{10}-\mathrm{C}_{11}$ & 1.398 & 1.399 & $\mathrm{C}_{42}-\mathrm{C}_{43}$ & 1.529 & \\
\hline $\mathrm{C}_{10}-\mathrm{H}_{12}$ & 1.081 & 0.930 & $\mathrm{C}_{42}-\mathrm{O}_{48}$ & 1.418 & \\
\hline $\mathrm{C}_{11}-\mathrm{O}_{35}$ & 1.368 & & $\mathrm{C}_{42}-\mathrm{H}_{63}$ & 1.100 & \\
\hline $\mathrm{O}_{14}-\mathrm{H}_{16}$ & 1.685 & & $\mathrm{C}_{43}-\mathrm{C}_{45}$ & 1.550 & \\
\hline $\mathrm{O}_{15}-\mathrm{H}_{16}$ & 0.995 & 0.910 & $\mathrm{C}_{43}-\mathrm{O}_{50}$ & 1.422 & \\
\hline $\mathrm{C}_{17}-\mathrm{C}_{18}$ & 1.399 & 1.389 & $\mathrm{C}_{43}-\mathrm{H}_{62}$ & 1.101 & \\
\hline $\mathrm{C}_{17}-\mathrm{C}_{19}$ & 1.400 & 1.392 & $\mathrm{C}_{44}-\mathrm{C}_{45}$ & 1.539 & \\
\hline $\mathrm{C}_{18}-\mathrm{C}_{20}$ & 1.393 & 1.383 & $\mathrm{C}_{44}-\mathrm{O}_{46}$ & 1.437 & \\
\hline $\mathrm{C}_{18}-\mathrm{H}_{21}$ & 1.085 & 0.930 & $\mathrm{C}_{44}-\mathrm{H}_{60}$ & 1.100 & \\
\hline $\mathrm{C}_{19}-\mathrm{C}_{22}$ & 1.391 & 1.377 & $\mathrm{C}_{44}-\mathrm{C}_{69}$ & 1.519 & \\
\hline $\mathrm{C}_{19}-\mathrm{H}_{23}$ & 1.087 & 0.930 & $\mathrm{C}_{45}-\mathrm{O}_{52}$ & 1.424 & \\
\hline $\mathrm{C}_{20}-\mathrm{C}_{24}$ & 1.400 & 1.385 & $\mathrm{C}_{45}-\mathrm{H}_{61}$ & 1.099 & \\
\hline $\mathrm{C}_{20}-\mathrm{H}_{25}$ & 1.088 & 0.930 & $\mathrm{O}_{48}-\mathrm{H}_{49}$ & 0.978 & \\
\hline $\mathrm{C}_{22}-\mathrm{C}_{24}$ & 1.399 & 1.376 & $\mathrm{O}_{50}-\mathrm{H}_{51}$ & 0.970 & \\
\hline $\mathrm{C}_{22}-\mathrm{H}_{26}$ & 1.085 & 0.930 & $\mathrm{O}_{52}-\mathrm{H}_{53}$ & 0.966 & \\
\hline $\mathrm{C}_{24}-\mathrm{O}_{27}$ & 1.365 & & $\mathrm{C}_{54}-\mathrm{O}_{55}$ & 1.412 & \\
\hline $\mathrm{O}_{27}-\mathrm{H}_{28}$ & 0.966 & & $\mathrm{C}_{54}-\mathrm{H}_{57}$ & 1.103 & \\
\hline $\mathrm{C}_{29}-\mathrm{C}_{30}$ & 1.549 & & $\mathrm{C}_{54}-\mathrm{H}_{58}$ & 1.095 & \\
\hline $\mathrm{C}_{29}-\mathrm{O}_{34}$ & 1.410 & & $\mathrm{O}_{55}-\mathrm{H}_{56}$ & 0.968 & \\
\hline
\end{tabular}




\begin{tabular}{|c|c|c|c|c|}
\hline $\mathrm{C}_{29}-\mathrm{O}_{35}$ & 1.412 & & $\mathrm{C}_{69}-\mathrm{H}_{70}$ & 1.095 \\
\hline Bond lengths (Å) & & & Bond lengths (Å) & \\
\hline $\mathrm{C}_{29}-\mathrm{H}_{67}$ & 1.096 & & $\mathrm{C}_{69}-\mathrm{H}_{71}$ & 1.092 \\
\hline $\mathrm{C}_{30}-\mathrm{C}_{31}$ & 1.534 & & $\mathrm{C}_{69}-\mathrm{H}_{72}$ & 1.093 \\
\hline Bond Angles ( ${ }^{\circ}$ ) & & & Bond Angles ( () & \\
\hline $\mathrm{C}_{5}-\mathrm{C}_{1}-\mathrm{O}_{13}$ & 109.96 & 120.85 & $\mathrm{C}_{17}-\mathrm{C}_{19}-\mathrm{C}_{22}$ & 121.24 \\
\hline $\mathrm{C}_{5}-\mathrm{C}_{1}-\mathrm{C}_{17}$ & 113.40 & 128.80 & $\mathrm{C}_{17}-\mathrm{C}_{19}-\mathrm{H}_{23}$ & 119.67 \\
\hline $\mathrm{C}_{5}-\mathrm{C}_{1}-\mathrm{H}_{73}$ & 108.54 & & $\mathrm{C}_{22}-\mathrm{C}_{19}-\mathrm{H}_{23}$ & 119.09 \\
\hline $\mathrm{O}_{13}-\mathrm{C}_{1}-\mathrm{C}_{17}$ & 108.05 & 111.15 & $\mathrm{C}_{18}-\mathrm{C}_{20}-\mathrm{C}_{24}$ & 120.00 \\
\hline $\mathrm{O}_{13}-\mathrm{C}_{1}-\mathrm{H}_{73}$ & 107.39 & & $\mathrm{C}_{18}-\mathrm{C}_{20}-\mathrm{H}_{25}$ & 120.02 \\
\hline $\mathrm{C}_{17}-\mathrm{C}_{1}-\mathrm{H}_{73}$ & 109.33 & & $\mathrm{C}_{24}-\mathrm{C}_{20}-\mathrm{H}_{25}$ & 119.98 \\
\hline $\mathrm{C}_{3}-\mathrm{C}_{2}-\mathrm{C}_{7}$ & 121.19 & 121.70 & $\mathrm{C}_{19}-\mathrm{C}_{22}-\mathrm{C}_{24}$ & 119.62 \\
\hline $\mathrm{C}_{3}-\mathrm{C}_{2}-\mathrm{O}_{13}$ & 121.41 & 122.03 & $\mathrm{C}_{19}-\mathrm{C}_{22}-\mathrm{H}_{26}$ & 121.37 \\
\hline $\mathrm{C}_{7}-\mathrm{C}_{2}-\mathrm{O}_{13}$ & 117.40 & 116.03 & $\mathrm{C}_{24}-\mathrm{C}_{22}-\mathrm{H}_{26}$ & 119.01 \\
\hline $\mathrm{C}_{2}-\mathrm{C}_{3}-\mathrm{C}_{4}$ & 120.71 & 119.12 & $\mathrm{C}_{20}-\mathrm{C}_{24}-\mathrm{C}_{22}$ & 119.78 \\
\hline $\mathrm{C}_{2}-\mathrm{C}_{3}-\mathrm{C}_{8}$ & 118.46 & 118.52 & $\mathrm{C}_{20}-\mathrm{C}_{24}-\mathrm{O}_{27}$ & 122.78 \\
\hline $\mathrm{C}_{4}-\mathrm{C}_{3}-\mathrm{C}_{8}$ & 120.66 & 122.33 & $\mathrm{C}_{22}-\mathrm{C}_{24}-\mathrm{O}_{27}$ & 117.44 \\
\hline $\mathrm{C}_{3}-\mathrm{C}_{4}-\mathrm{C}_{5}$ & 115.78 & 115.93 & $\mathrm{C}_{24}-\mathrm{O}_{27}-\mathrm{H}_{28}$ & 109.21 \\
\hline $\mathrm{C}_{3}-\mathrm{C}_{4}-\mathrm{O}_{14}$ & 123.06 & & $\mathrm{C}_{30}-\mathrm{C}_{29}-\mathrm{O}_{34}$ & 112.11 \\
\hline $\mathrm{C}_{5}-\mathrm{C}_{4}-\mathrm{O}_{14}$ & 121.13 & & $\mathrm{C}_{30}-\mathrm{C}_{29}-\mathrm{O}_{35}$ & 107.21 \\
\hline $\mathrm{C}_{1}-\mathrm{C}_{5}-\mathrm{C}_{4}$ & 111.08 & 122.03 & $\mathrm{C}_{30}-\mathrm{C}_{29}-\mathrm{H}_{67}$ & 109.79 \\
\hline $\mathrm{C}_{1}-\mathrm{C}_{5}-\mathrm{H}_{9}$ & 109.46 & & $\mathrm{O}_{34}-\mathrm{C}_{29}-\mathrm{O}_{35}$ & 112.95 \\
\hline $\mathrm{C}_{1}-\mathrm{C}_{5}-\mathrm{H}_{68}$ & 110.97 & & $\mathrm{O}_{34}-\mathrm{C}_{29}-\mathrm{H}_{67}$ & 104.98 \\
\hline $\mathrm{C}_{4}-\mathrm{C}_{5}-\mathrm{H}_{9}$ & 108.41 & & $\mathrm{O}_{35}-\mathrm{C}_{29}-\mathrm{H}_{67}$ & 109.80 \\
\hline $\mathrm{C}_{4}-\mathrm{C}_{5}-\mathrm{H}_{68}$ & 109.43 & & $\mathrm{C}_{29}-\mathrm{C}_{30}-\mathrm{C}_{31}$ & 108.12 \\
\hline $\mathrm{H}_{9}-\mathrm{C}_{5}-\mathrm{H}_{68}$ & 107.38 & & $\mathrm{C}_{29}-\mathrm{C}_{30}-\mathrm{O}_{40}$ & 112.41 \\
\hline $\mathrm{C}_{2}-\mathrm{C}_{7}-\mathrm{C}_{6}$ & 121.16 & & $\mathrm{C}_{29}-\mathrm{C}_{30}-\mathrm{H}_{59}$ & 108.86 \\
\hline $\mathrm{C}_{2}-\mathrm{C}_{7}-\mathrm{C}_{11}$ & 118.76 & 118.72 & $\mathrm{C}_{31}-\mathrm{C}_{30}-\mathrm{O}_{40}$ & 107.36 \\
\hline $\mathrm{C}_{6}-\mathrm{C}_{7}-\mathrm{C}_{11}$ & 120.06 & & $\mathrm{C}_{31}-\mathrm{C}_{30}-\mathrm{H}_{59}$ & 110.16 \\
\hline $\mathrm{C}_{3}-\mathrm{C}_{8}-\mathrm{C}_{10}$ & 120.74 & 120.20 & $\mathrm{O}_{40}-\mathrm{C}_{30}-\mathrm{H}_{59}$ & 109.91 \\
\hline $\mathrm{C}_{3}-\mathrm{C}_{8}-\mathrm{O}_{15}$ & 120.54 & 121.20 & $\mathrm{C}_{30}-\mathrm{C}_{31}-\mathrm{C}_{33}$ & 108.94 \\
\hline $\mathrm{C}_{10}-\mathrm{C}_{8}-\mathrm{O}_{15}$ & 118.71 & 116.80 & $\mathrm{C}_{30}-\mathrm{C}_{31}-\mathrm{O}_{38}$ & 111.22 \\
\hline $\mathrm{C}_{8}-\mathrm{C}_{10}-\mathrm{C}_{11}$ & 118.63 & 120.35 & $\mathrm{C}_{30}-\mathrm{C}_{31}-\mathrm{H}_{64}$ & 109.10 \\
\hline $\mathrm{C}_{8}-\mathrm{C}_{10}-\mathrm{H}_{12}$ & 118.62 & & $\mathrm{C}_{33}-\mathrm{C}_{31}-\mathrm{O}_{38}$ & 109.57 \\
\hline $\mathrm{C}_{11}-\mathrm{C}_{10}-\mathrm{H}_{12}$ & 122.70 & & $\mathrm{C}_{33}-\mathrm{C}_{31}-\mathrm{H}_{64}$ & 109.72 \\
\hline $\mathrm{C}_{7}-\mathrm{C}_{11}-\mathrm{C}_{10}$ & 122.22 & 120.48 & $\mathrm{O}_{38}-\mathrm{C}_{31}-\mathrm{H}_{64}$ & 108.27 \\
\hline $\mathrm{C}_{7}-\mathrm{C}_{11}-\mathrm{O}_{35}$ & 114.01 & & $\mathrm{C}_{33}-\mathrm{C}_{32}-\mathrm{O}_{34}$ & 109.67 \\
\hline $\mathrm{C}_{10}-\mathrm{C}_{11}-\mathrm{O}_{35}$ & 123.77 & & $\mathrm{C}_{33}-\mathrm{C}_{32}-\mathrm{C}_{54}$ & 113.78 \\
\hline
\end{tabular}




\begin{tabular}{|c|c|c|c|c|}
\hline $\mathrm{C}_{1}-\mathrm{O}_{13}-\mathrm{C}_{2}$ & 116.59 & 120.85 & $\mathrm{C}_{33}-\mathrm{C}_{32}-\mathrm{H}_{66}$ & 109.20 \\
\hline $\mathrm{C}_{8}-\mathrm{O}_{15}-\mathrm{H}_{16}$ & 106.52 & & $\mathrm{O}_{34}-\mathrm{C}_{32}-\mathrm{C}_{54}$ & 105.99 \\
\hline Bond Angles ( ${ }^{\circ}$ ) & & & Bond Angles ( $\left(^{\circ}\right)$ & \\
\hline $\mathrm{C}_{1}-\mathrm{C}_{17}-\mathrm{C}_{18}$ & 121.16 & & $\mathrm{O}_{34}-\mathrm{C}_{32}-\mathrm{H}_{66}$ & 109.00 \\
\hline $\mathrm{C}_{1}-\mathrm{C}_{17}-\mathrm{C}_{19}$ & 120.24 & 122.71 & $\mathrm{C}_{54}-\mathrm{C}_{32}-\mathrm{H}_{66}$ & 109.07 \\
\hline $\mathrm{C}_{18}-\mathrm{C}_{17}-\mathrm{C}_{19}$ & 118.55 & 117.19 & $\mathrm{C}_{31}-\mathrm{C}_{33}-\mathrm{C}_{32}$ & 110.36 \\
\hline $\mathrm{C}_{17}-\mathrm{C}_{18}-\mathrm{C}_{20}$ & 120.80 & 119.30 & $\mathrm{C}_{31}-\mathrm{C}_{33}-\mathrm{O}_{36}$ & 109.48 \\
\hline $\mathrm{C}_{17}-\mathrm{C}_{18}-\mathrm{H}_{21}$ & 119.55 & & $\mathrm{C}_{31}-\mathrm{C}_{33}-\mathrm{H}_{65}$ & 109.20 \\
\hline $\mathrm{C}_{20}-\mathrm{C}_{18}-\mathrm{H}_{21}$ & 119.64 & & $\mathrm{C}_{32}-\mathrm{C}_{33}-\mathrm{O}_{36}$ & 111.44 \\
\hline $\mathrm{C}_{32}-\mathrm{C}_{33}-\mathrm{H}_{65}$ & 110.90 & & $\mathrm{C}_{32}-\mathrm{C}_{54}-\mathrm{O}_{55}$ & 112.01 \\
\hline $\mathrm{O}_{36}-\mathrm{C}_{33}-\mathrm{H}_{65}$ & 105.31 & & $\mathrm{C}_{32}-\mathrm{C}_{54}-\mathrm{H}_{57}$ & 108.61 \\
\hline $\mathrm{C}_{29}-\mathrm{O}_{34}-\mathrm{C}_{32}$ & 114.52 & & $\mathrm{C}_{32}-\mathrm{C}_{54}-\mathrm{H}_{58}$ & 108.86 \\
\hline $\mathrm{C}_{11}-\mathrm{O}_{35}-\mathrm{C}_{29}$ & 119.78 & & $\mathrm{O}_{55}-\mathrm{C}_{54}-\mathrm{H}_{57}$ & 111.97 \\
\hline $\mathrm{C}_{33}-\mathrm{O}_{36}-\mathrm{H}_{37}$ & 105.12 & & $\mathrm{O}_{55}-\mathrm{C}_{54}-\mathrm{H}_{58}$ & 107.36 \\
\hline $\mathrm{C}_{31}-\mathrm{O}_{38}-\mathrm{H}_{39}$ & 104.36 & & $\mathrm{H}_{57}-\mathrm{C}_{54}-\mathrm{H}_{58}$ & 107.91 \\
\hline $\mathrm{C}_{30}-\mathrm{O}_{40}-\mathrm{C}_{41}$ & 116.34 & & $\mathrm{C}_{54}-\mathrm{O}_{55}-\mathrm{H}_{56}$ & 106.57 \\
\hline $\mathrm{O}_{40}-\mathrm{C}_{41}-\mathrm{C}_{42}$ & 108.94 & & $\mathrm{C}_{44}-\mathrm{C}_{69}-\mathrm{H}_{70}$ & 110.15 \\
\hline $\mathrm{O}_{40}-\mathrm{C}_{41}-\mathrm{O}_{46}$ & 108.38 & & $\mathrm{C}_{44}-\mathrm{C}_{69}-\mathrm{H}_{71}$ & 109.57 \\
\hline $\mathrm{O}_{40}-\mathrm{C}_{41}-\mathrm{H}_{47}$ & 107.95 & & $\mathrm{C}_{44}-\mathrm{C}_{69}-\mathrm{H}_{72}$ & 110.51 \\
\hline $\mathrm{C}_{42}-\mathrm{C}_{41}-\mathrm{O}_{46}$ & 113.73 & & $\mathrm{H}_{70}-\mathrm{C}_{69}-\mathrm{H}_{71}$ & 109.00 \\
\hline $\mathrm{C}_{42}-\mathrm{C}_{41}-\mathrm{H}_{47}$ & 109.52 & & $\mathrm{H}_{70}-\mathrm{C}_{69}-\mathrm{H}_{72}$ & 108.38 \\
\hline $\mathrm{O}_{46}-\mathrm{C}_{41}-\mathrm{H}_{47}$ & 108.15 & & $\mathrm{H}_{71}-\mathrm{C}_{69}-\mathrm{H}_{72}$ & 109.20 \\
\hline $\mathrm{C}_{41}-\mathrm{C}_{42}-\mathrm{C}_{43}$ & 110.15 & & $\mathrm{C}_{32}-\mathrm{C}_{54}-\mathrm{O}_{55}$ & 112.01 \\
\hline $\mathrm{C}_{41}-\mathrm{C}_{42}-\mathrm{O}_{48}$ & 110.63 & & $\mathrm{C}_{32}-\mathrm{C}_{54}-\mathrm{H}_{57}$ & 108.61 \\
\hline $\mathrm{C}_{41}-\mathrm{C}_{42}-\mathrm{H}_{63}$ & 108.33 & & $\mathrm{C}_{32}-\mathrm{C}_{54}-\mathrm{H}_{58}$ & 108.86 \\
\hline $\mathrm{C}_{43}-\mathrm{C}_{42}-\mathrm{O}_{48}$ & 107.85 & & $\mathrm{O}_{55}-\mathrm{C}_{54}-\mathrm{H}_{57}$ & 111.97 \\
\hline $\mathrm{C}_{43}-\mathrm{C}_{42}-\mathrm{H}_{63}$ & 108.91 & & $\mathrm{O}_{55}-\mathrm{C}_{54}-\mathrm{H}_{58}$ & 107.36 \\
\hline $\mathrm{O}_{48}-\mathrm{C}_{42}-\mathrm{H}_{63}$ & 110.96 & & $\mathrm{H}_{57}-\mathrm{C}_{54}-\mathrm{H}_{58}$ & 107.91 \\
\hline $\mathrm{C}_{42}-\mathrm{C}_{43}-\mathrm{C}_{45}$ & 111.34 & & $\mathrm{C}_{54}-\mathrm{O}_{55}-\mathrm{H}_{56}$ & 106.57 \\
\hline $\mathrm{C}_{42}-\mathrm{C}_{43}{ }^{-}{ }_{50}$ & 109.69 & & $\mathrm{C}_{44}-\mathrm{C}_{69}-\mathrm{H}_{70}$ & 110.15 \\
\hline $\mathrm{C}_{42}-\mathrm{C}_{43}-\mathrm{H}_{62}$ & 108.26 & & $\mathrm{C}_{44}-\mathrm{C}_{69}-\mathrm{H}_{71}$ & 109.57 \\
\hline $\mathrm{C}_{45}-\mathrm{C}_{43}{ }^{-}{ }_{50}$ & 108.45 & & $\mathrm{C}_{44}-\mathrm{C}_{69}-\mathrm{H}_{72}$ & 110.51 \\
\hline $\mathrm{C}_{45}-\mathrm{C}_{43}-\mathrm{H}_{62}$ & 108.29 & & $\mathrm{H}_{70}-\mathrm{C}_{69}-\mathrm{H}_{71}$ & 109.00 \\
\hline${ }_{50}-\mathrm{C}_{43}-\mathrm{H}_{62}$ & 110.82 & & $\mathrm{H}_{70}-\mathrm{C}_{69}-\mathrm{H}_{72}$ & 108.38 \\
\hline $\mathrm{C}_{45}-\mathrm{C}_{44}-\mathrm{O}_{46}$ & 109.80 & & $\mathrm{H}_{71}-\mathrm{C}_{69}-\mathrm{H}_{72}$ & 109.20 \\
\hline $\mathrm{C}_{45}-\mathrm{C}_{44}-\mathrm{H}_{60}$ & 107.84 & & Dihedral angles $(\stackrel{\circ}{)})$ & \\
\hline $\mathrm{C}_{45}-\mathrm{C}_{44}-\mathrm{C}_{69}$ & 112.91 & & $\mathrm{O}_{13}-\mathrm{C}_{1}-\mathrm{C}_{5}-\mathrm{C}_{4}$ & -54.56 \\
\hline $\mathrm{O}_{46}-\mathrm{C}_{44}-\mathrm{H}_{60}$ & 110.48 & & $\mathrm{O}_{13}-\mathrm{C}_{1}-\mathrm{C}_{5}-\mathrm{H}_{9}$ & 65.13 \\
\hline
\end{tabular}




\begin{tabular}{|c|c|c|c|}
\hline $\mathrm{O}_{46}-\mathrm{C}_{44}-\mathrm{C}_{69}$ & 106.72 & $\mathrm{O}_{13}-\mathrm{C}_{1}-\mathrm{C}_{5}-\mathrm{H}_{68}$ & -176.53 \\
\hline $\mathrm{H}_{61}-\mathrm{C}_{44}-\mathrm{C}_{69}$ & 109.10 & $\mathrm{C}_{17}-\mathrm{C}_{1}-\mathrm{C}_{5}-\mathrm{C}_{4}$ & -175.65 \\
\hline $\mathrm{C}_{43}-\mathrm{C}_{45}-\mathrm{C}_{44}$ & 111.98 & $\mathrm{C}_{17}-\mathrm{C}_{1}-\mathrm{C}_{5}-\mathrm{H}_{9}$ & -55.96 \\
\hline Bond angles ( $\AA$ ) & & Dihedral angles $(\stackrel{\circ}{)})$ & \\
\hline $\mathrm{C}_{43}-\mathrm{C}_{45}-\mathrm{O}_{52}$ & 112.34 & $\mathrm{C}_{17}-\mathrm{C}_{1}-\mathrm{C}_{5}-\mathrm{H}_{68}$ & 62.38 \\
\hline $\mathrm{C}_{43}-\mathrm{C}_{45}-\mathrm{H}_{61}$ & 106.70 & $\mathrm{H}_{73}-\mathrm{C}_{1}-\mathrm{C}_{5}-\mathrm{C}_{4}$ & 62.65 \\
\hline $\mathrm{C}_{44}-\mathrm{C}_{45}-\mathrm{O}_{52}$ & 105.67 & $\mathrm{H}_{73}-\mathrm{C}_{1}-\mathrm{C}_{5}-\mathrm{H}_{9}$ & -177.67 \\
\hline $\mathrm{C}_{44}-\mathrm{C}_{45}-\mathrm{H}_{61}$ & 108.62 & $\mathrm{H}_{73}-\mathrm{C}_{1}-\mathrm{C}_{5}-\mathrm{H}_{68}$ & -59.33 \\
\hline $\mathrm{O}_{52}-\mathrm{C}_{45}-\mathrm{H}_{61}$ & 111.54 & $\mathrm{C}_{5}-\mathrm{C}_{1}-\mathrm{O}_{13}-\mathrm{C}_{2}$ & 51.63 \\
\hline $\mathrm{C}_{41}-\mathrm{O}_{46}-\mathrm{C}_{44}$ & 117.45 & $\mathrm{C}_{17}-\mathrm{C}_{1}-\mathrm{O}_{13}-\mathrm{C}_{2}$ & 175.88 \\
\hline $\mathrm{C}_{42}-\mathrm{O}_{48}-\mathrm{H}_{49}$ & 108.56 & $\mathrm{H}_{73}-\mathrm{C}_{1}-\mathrm{O}_{13}-\mathrm{C}_{2}$ & -66.28 \\
\hline $\mathrm{C}_{43}{ }^{-}-\mathrm{H}_{51}$ & 105.73 & $\mathrm{C}_{5}-\mathrm{C}_{1}-\mathrm{C}_{17}-\mathrm{C}_{18}$ & 80.31 \\
\hline $\mathrm{C}_{45}-\mathrm{O}_{52}-\mathrm{H}_{53}$ & 107.93 & $\mathrm{C}_{5}-\mathrm{C}_{1}-\mathrm{C}_{17}-\mathrm{C}_{19}$ & -97.28 \\
\hline
\end{tabular}

\section{Vibrational Assignments}

The Naringin molecule contains 73 atoms (including $8-\mathrm{OH}$ group, one methylene and methyl groups); hence it can have 213 normal modes of vibrations. To the best of our knowledge, there is no complete vibrational spectroscopic study carried out on Naringin. In the present investigation the FT-IR and FTRaman spectra were recorded in the region of $4000-400 \mathrm{~cm}^{-1}$ and $3500-50 \mathrm{~cm}^{-1}$, respectively. Stimulated IR and Raman spectra were constructed and compared with experimental spectra. The combined spectrum of FT-IR showed in Figure 2 and the FT-Raman spectrum showed in Figure 3. Similarly, Observed frequencies and calculated wave numbers are compared and listed in Table 1, also provided a detail results in Table S2 (Supporting information). The frequency calculation was performed by using B3LYP level of theory using 6-31G $(\mathrm{d}, \mathrm{p})$ basis set. The TED for all fundamental vibrations is calculated using SQM method.

\section{C-H Vibrations}

The heteroaromatic structure shows the presence of $\mathrm{C}-\mathrm{H}$ vibration in the region $3100-3000 \mathrm{~cm}^{-1}$, which is the characteristic region for the ready identification of $\mathrm{C}-\mathrm{H}$ stretching vibration [18]. In this region, the bands are not affected appreciably by nature of the substituent. In the present work, the $\mathrm{C}-\mathrm{H}$ aromatic stretching frequency observed at $3071 \mathrm{~cm}^{-1}$ as a weak band in FT-Raman and its corresponding calculated wavenumber is $3083 \mathrm{~cm}^{-1}$ (mode numbers: 202). For the same mode five more harmonic vibrations are also appeared (mode numbers: 200, 201, 203-205). As expected these modes are pure stretching modes as it evident from the TED column (their contribution 95\%). The FT-IR bands $2890-2970 \mathrm{~cm}^{-1}$ (weak) and FT-Raman bands 2939 , 2958, $2976 \mathrm{~cm}^{-1}$ (weak) are assigned to C-H stretching in ring B and ring D respectively. These assignments are comparable with harmonic frequencies 2888, 2939, 2943, 2952, 2965 and 2985 $\mathrm{cm}^{-1}$ (mode numbers: 185, 191, 193-196) and also find support from TED. The mode numbers: 183, 184, 186, 188 and 192 are

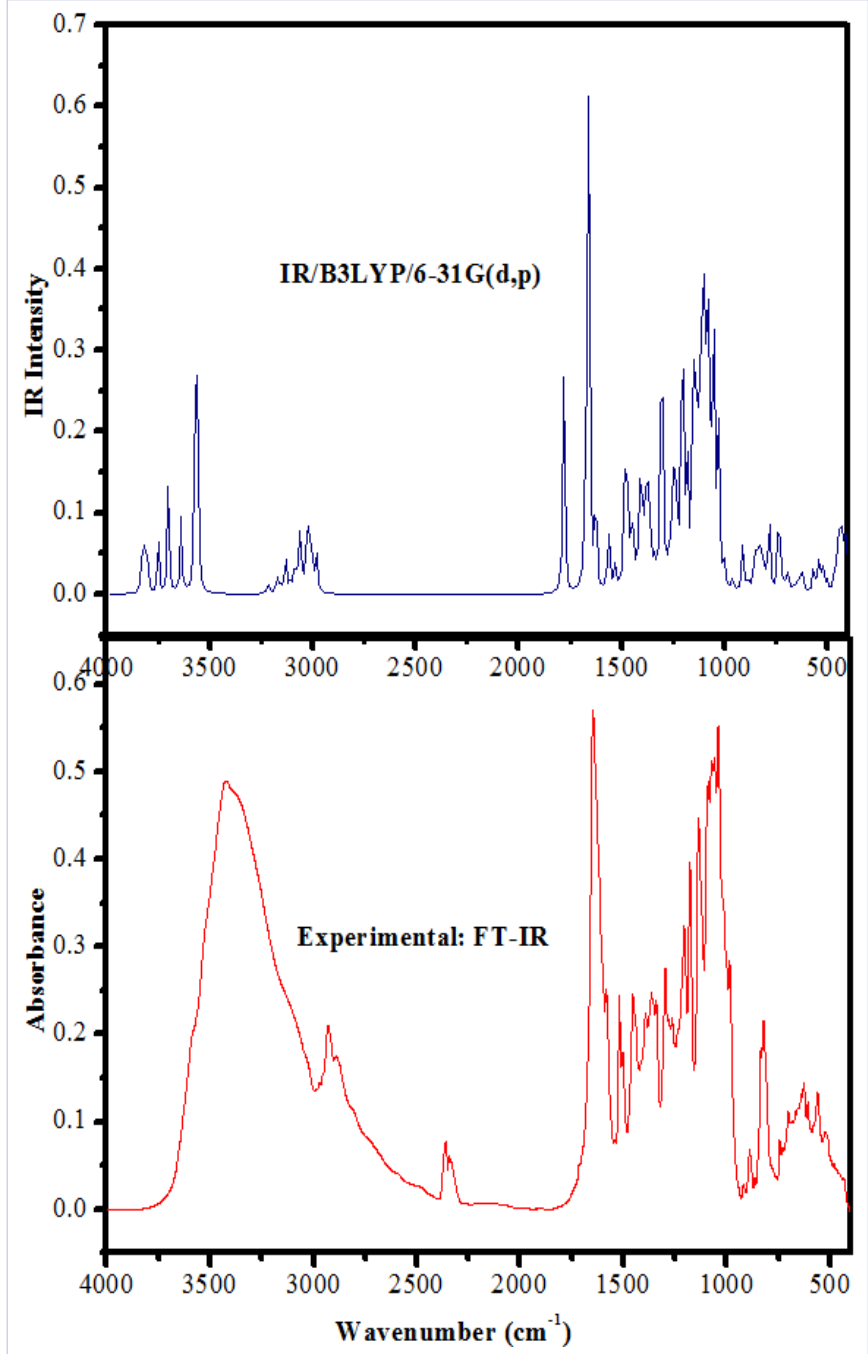

Figure 2: The combined experimental and theoretical FT-IR spectra of Naringin. 


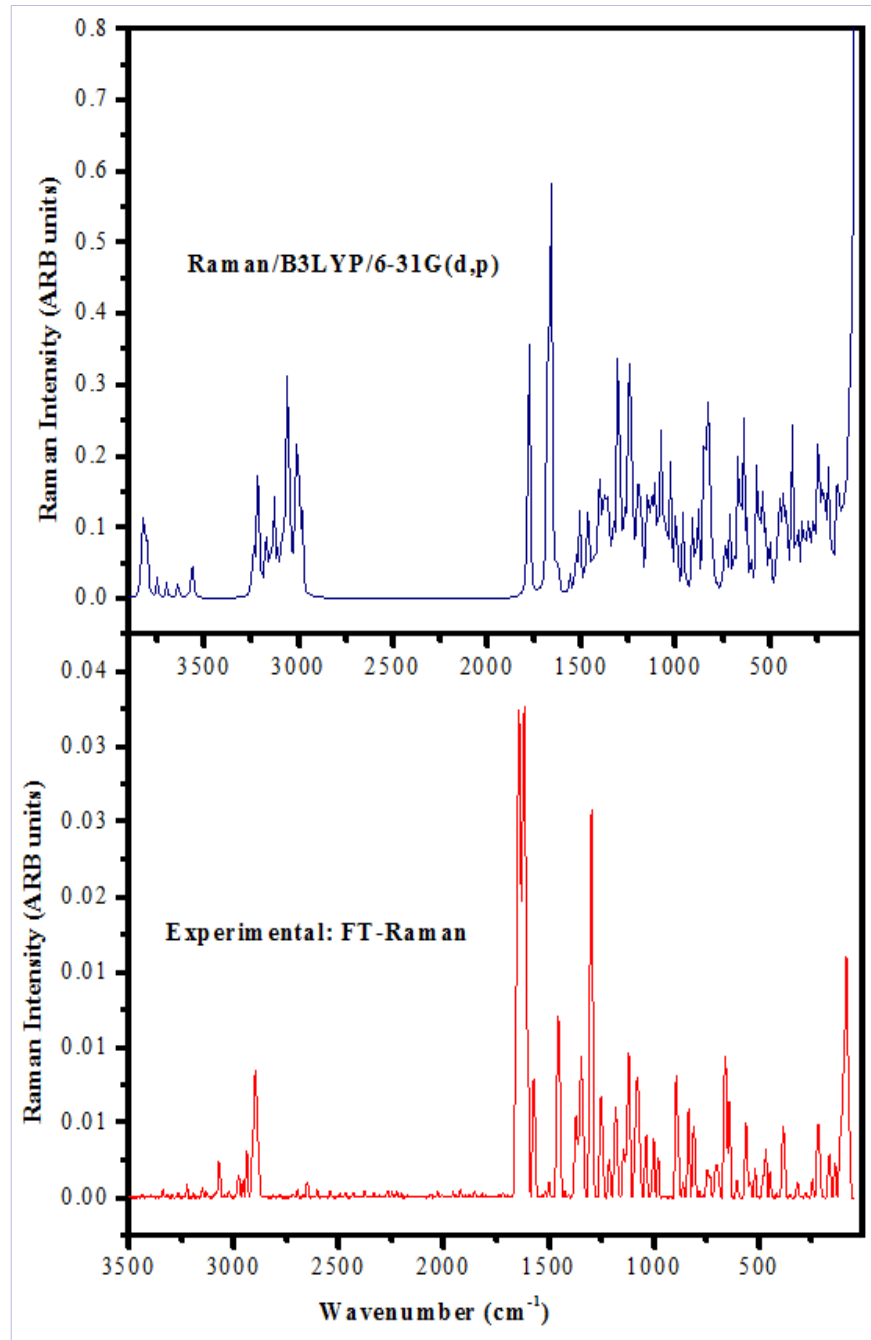

Figure 3: The combined experimental and theoretical FT-Raman spectra of Naringin. assigned to $v_{\mathrm{C}-\mathrm{H}}$ in ring $\mathrm{E}$. The in-plane bending of $\mathrm{C}-\mathrm{H}$ is observed at 1089, 1178 and 1296 (strong) in FT-IR and $1002 \mathrm{~cm}^{-1}$ in FTRaman spectra. The calculated wavenumbers in the range 990 $1312 \mathrm{~cm}^{-1}$ (mode numbers: 101, 117, 125-128, 140, 143, 145147 ) are assigned to $\delta_{C-H}$ with considerable TED value. The Outof-plane bending of $\tau_{\mathrm{C}-\mathrm{H}}$ mode is observed at $896,812,607 \mathrm{~cm}^{-1}$ in FT-Raman and 919 and $608 \mathrm{~cm}^{-1}$ in FT-IR spectra, whereas the harmonic values are in the range of $605-922 \mathrm{~cm}^{-1}$ (mode nos: 67 , $68,80-82,86,94,95)$. These assignments are in agreement with literature values [18-19].

\section{Methyl Group $\left(\mathrm{CH}_{3}\right)$ Vibrations}

The $\mathrm{C}$ - $\mathrm{H}$ stretching mode in $\mathrm{CH}_{3}$ occurs at lower wavenumber than those of the aromatic ring (3000-3100 $\left.\mathrm{cm}^{-1}\right)$. The asymmetric stretching mode of the $\mathrm{CH}_{3}$ group is expected to occur in the region of $2980 \mathrm{~cm}^{-1}$ and the symmetric one is at $2870 \mathrm{~cm}^{-1}$ [2021]. The methyl group $\left(\mathrm{CH}_{3}\right)$ has three stretching vibrations, namely one symmetric and two asymmetric modes [22]. In this study, the computed wavenumbers for the $\mathrm{CH}_{3}$ (asy) stretching and $\mathrm{CH}_{3}$ (sym) stretching are $3021,3008 \mathrm{~cm}^{-1}$ (mode numbers: 199, 198) and $2938 \mathrm{~cm}^{-1}$ /mode no: 190, respectively. These modes are supported by TED and above literature. These modes are $100 \%$ (TED) pure for $\mathrm{CH}_{3}$ stretching vibration.

Two asymmetric $\mathrm{CH}_{3}$ bending, one symmetric $\mathrm{CH}_{3}$ bending, two $\mathrm{CH}_{3}$ rocking and one $\mathrm{CH}_{3}$ torsional vibrations are possible [22]. The methyl group symmetric deformation absorbs moderately to strong in the range $1365 \pm 25 \mathrm{~cm}^{-1}$ and asymmetric methyl deformations in the region 1390-1480 $\mathrm{cm}^{-1}$ [23]. Based on the above conclusion the observed bands 1453/1456 (FT-IR/ Raman) and $1363 \mathrm{~cm}^{-1} /$ FT-IR are attributed to $\delta \mathrm{CH}_{3}$ (asym) and $\delta \mathrm{CH}_{3}$ (sym), respectively. The methyl rocking generally appears in the region $1050 \pm 30$ and $975+45 \mathrm{~cm}^{-1}[24]$, as a weak moderate or sometimes strong band, the wavenumber of which is coupled to the C-C stretching vibrations, which occur in the neighborhood of $900 \mathrm{~cm}^{-1}$. The FT-Raman band $1122 \mathrm{~cm}^{-1}$ (medium strong) and

Table S2: The vibrational assignments of Naringin using Scaled Quantum Mechanics method (B3LYP/6-31G(d,p)).

\begin{tabular}{|c|c|c|c|c|c|c|}
\hline Mode No. & Scaled B3LYPa & FT-IR & FT-Raman & $I_{I R}^{b}$ & $I_{\text {Raman }}^{c}$ & Vibrational assignments TED $^{d}(\geq 10)$ \\
\hline 1 & 3 & & & 0.05 & 79.30 & $\tau_{\mathrm{C} 29-035-\mathrm{C} 11-\mathrm{C} 7}(46)+\tau_{\mathrm{C} 29-035-\mathrm{C} 11-\mathrm{C} 10}(45)$ \\
\hline 2 & 14 & & & 0.00 & 13.63 & $\tau_{\mathrm{C} 32-034-\mathrm{C} 29-035}(11)+\tau_{\mathrm{C} 41-040-\mathrm{C} 30-\mathrm{C} 29}(14)$ \\
\hline 3 & 18 & & & 0.02 & 28.67 & $\tau_{\mathrm{C} 11-035-\mathrm{C} 29-\mathrm{C} 30}(21)+\tau_{\mathrm{C} 11-035-\mathrm{C} 29-034}(18)$ \\
\hline 4 & 23 & & & 0.13 & 100 & $\tau_{\mathrm{C} 18-\mathrm{C} 17-\mathrm{C} 1-\mathrm{O} 13 \mathrm{O}}(15)+\tau_{\mathrm{C} 19-\mathrm{C} 17-\mathrm{C} 1-\mathrm{C} 5}(23)+\Gamma_{\mathrm{C} 19-\mathrm{C} 17-}$ \\
\hline 5 & 33 & & & 0.01 & 6.17 & $\tau_{\mathrm{C} 42-\mathrm{C} 41-040-\mathrm{C} 30}(10)$ \\
\hline 6 & 34 & & & 0.13 & 17.21 & $\tau_{\mathrm{CCCC}}(3)$ \\
\hline 7 & 38 & & & 0.10 & 22.75 & $\tau_{\mathrm{COCC}}(30)$ \\
\hline 8 & 39 & & & 0.16 & 20.95 & $\tau_{\mathrm{C} 2-013-\mathrm{C} 1-\mathrm{C} 17}(22)$ \\
\hline 9 & 50 & & & 0.04 & 3.72 & $\tau_{\mathrm{C} 44-046-\mathrm{C} 41-040}(16)$ \\
\hline 10 & 60 & & & 0.25 & 4.79 & $\tau_{\mathrm{CCCC}}(10)+\tau_{\mathrm{ocCC}}(13)$ \\
\hline 11 & 76 & & & 0.02 & 2.30 & $\tau_{\mathrm{OCOC}}(10)$ \\
\hline
\end{tabular}




\begin{tabular}{|c|c|c|c|c|c|}
\hline 12 & 82 & $86 \mathrm{~s}$ & 0.37 & 1.09 & $\tau \operatorname{cccc}(16)+\Gamma \operatorname{occc}(13)$ \\
\hline 13 & 95 & & 0.09 & 1.15 & $\delta_{\mathrm{C} 11-035-\mathrm{C} 29}(10)+\tau_{\mathrm{C} 41-040-\mathrm{C} 30-\mathrm{C} 31}(10)$ \\
\hline 14 & 103 & & 0.29 & 1.01 & $\tau_{\text {oссо }}(10)$ \\
\hline 15 & 111 & & 0.62 & 0.54 & $\tau_{\mathrm{occc}}(13)$ \\
\hline 16 & 114 & & 0.46 & 0.70 & $\tau_{055 \mathrm{C54C32С33}}(10)$ \\
\hline 17 & 126 & & 1.04 & 1.01 & $\delta_{\text {C41-040-C30 }}(12)+\tau_{\text {C44-046-C41-040 }}(11)$ \\
\hline 18 & 132 & $140 w$ & 0.14 & 2.02 & $\tau \operatorname{ccco}(10)$ \\
\hline 19 & 143 & & 0.34 & 2.22 & $\tau_{\mathrm{occC}}(10)$ \\
\hline 20 & 169 & $168 w$ & 0.50 & 1.30 & $\delta \operatorname{ccc}(15)$ \\
\hline 21 & 182 & & 0.21 & 1.36 & $v_{\mathrm{cc}}(10)+\delta_{\mathrm{ccC}}(13)+\tau_{\mathrm{occc}}(10)$ \\
\hline 22 & 186 & & 2.01 & 2.70 & $\delta_{\text {ocC }}(10)$ \\
\hline 23 & 206 & & 3.04 & 2.61 & $\delta_{\text {ocC }}(10)$ \\
\hline 24 & 209 & & 0.08 & 0.99 & $\tau_{\mathrm{HCCC}}(37)+\tau_{\mathrm{HCCO}}(18)+\tau_{\mathrm{HCCH}}(24)$ \\
\hline 25 & 215 & & 0.36 & 0.57 & $\tau_{\mathrm{C} 11-\mathrm{C} 10-\mathrm{C} 8-015}(20)$ \\
\hline 26 & 220 & $220 w$ & 1.31 & 0.59 & $\delta_{\mathrm{C} 45-\mathrm{C} 44-\mathrm{C} 69}(14)+\tau_{\mathrm{HCCC}}(10)$ \\
\hline 27 & 228 & & 1.85 & 2.90 & $\tau_{\mathrm{H} 56055 \mathrm{C} 54 \mathrm{C} 32}(10)$ \\
\hline 28 & 229 & & 0.72 & 0.55 & $\tau_{052 C 45 C 44}(10)$ \\
\hline 29 & 240 & $247 w$ & 8.53 & 3.46 & $\tau_{\mathrm{H} 56-055-\mathrm{C5} 5-\mathrm{C} 32}(32)+\tau_{\mathrm{H} 56-055-\mathrm{C} 54-\mathrm{H} 57}(22)+\tau_{\mathrm{H} 56-055-}$ \\
\hline 30 & 256 & & 0.40 & 1.88 & $\delta_{\mathrm{cCC}}(10)$ \\
\hline 31 & 267 & & 0.80 & 0.96 & $\tau_{\text {носс }}(10)$ \\
\hline 32 & 283 & & 0.36 & 0.91 & $\delta_{\mathrm{ccC}}(10)+\delta_{\mathrm{occ}}(10)$ \\
\hline 33 & 284 & & 0.78 & 1.82 & $\tau_{\mathrm{C} 41040 \mathrm{C} 30}(10)$ \\
\hline 34 & 293 & & 0.48 & 0.62 & $\tau_{\text {038с31с30040 }}(10)$ \\
\hline 35 & 300 & & 0.94 & 0.77 & $\tau_{\mathrm{occC}}(10)+\Gamma_{\mathrm{occo}}(10)$ \\
\hline 36 & 309 & & 0.43 & 0.82 & $\delta_{055 \mathrm{C} 54 \mathrm{C} 32}(16)$ \\
\hline 37 & 315 & & 0.10 & 1.29 & $\delta_{\text {ocC }}(10)$ \\
\hline 38 & 321 & & 2.60 & 0.71 & $\delta_{\text {occ }}(15)+\delta_{\text {сco }}(10)$ \\
\hline 39 & 340 & & 15.04 & 2.29 & $\tau_{\mathrm{H} 28-027-\mathrm{C} 24-\mathrm{C} 20}(47)+\tau_{\mathrm{H} 28-027-\mathrm{C} 24-\mathrm{C} 22}(52)$ \\
\hline 40 & 345 & & 0.70 & 0.27 & $\delta_{\text {осс }}(14)+\delta_{\text {ссо }}(10)+\tau_{\text {носс }}(11)$ \\
\hline 41 & 363 & & 2.62 & 1.00 & $\delta_{\mathrm{ocC}}(11)+\tau_{\mathrm{occc}}(13)$ \\
\hline 42 & 365 & & 12.40 & 1.06 & $\tau_{\mathrm{H} 53-052-\mathrm{C} 45-\mathrm{C} 43}(29)+\tau_{\mathrm{H} 53-052-\mathrm{C} 45-\mathrm{C} 44}(17)+\tau_{\mathrm{H} 53-052-}$ \\
\hline 43 & 366 & & 0.69 & 2.83 & $\delta_{\text {ocC }}(10)$ \\
\hline 44 & 376 & $385 w$ & 0.98 & 0.86 & $\delta_{\text {occ }}(10)$ \\
\hline 45 & 395 & & 6.04 & 1.46 & $\tau_{\mathrm{H} 51-050-\mathrm{C} 43-\mathrm{C} 45}(14)$ \\
\hline 46 & 399 & & 4.05 & 0.83 & $\delta_{027-\mathrm{C} 24-\mathrm{C} 20}(20)+\delta_{027-\mathrm{C} 24-\mathrm{C} 22}(23)$ \\
\hline 47 & 402 & & 2.25 & 0.27 & $\delta_{027-\mathrm{C} 24-\mathrm{C} 20}(11)$ \\
\hline 48 & 406 & & 0.19 & 0.29 & $\tau_{\mathrm{C} 24-\mathrm{C} 20-\mathrm{C} 18-\mathrm{C} 17}(18)+\tau_{\mathrm{C} 24-\mathrm{C} 22-\mathrm{C} 19-\mathrm{C} 17}(18)$ \\
\hline 49 & 409 & & 3.30 & 1.23 & $\tau_{\mathrm{H} 51-050-\mathrm{C} 43-\mathrm{C} 42}(11)+\tau \Gamma_{\mathrm{H} 51-050-\mathrm{C} 43-\mathrm{H} 62}(12)$ \\
\hline
\end{tabular}




\begin{tabular}{|c|c|c|c|c|c|c|}
\hline 50 & 417 & & & 4.16 & 1.81 & $\tau_{\mathrm{H} 51-050-\mathrm{C} 43-\mathrm{C} 42}(15)$ \\
\hline 51 & 419 & & & 11.62 & 1.14 & $\tau_{\mathrm{H} 16-015-\mathrm{c} 8-\mathrm{cc}}(31)+\tau_{\mathrm{H} 16-015-\mathrm{c8}-\mathrm{c} 10}(32)$ \\
\hline 52 & 432 & & & 0.49 & 1.84 & $\tau_{\text {носс }}(13)$ \\
\hline 53 & 435 & $434 w$ & & 5.92 & 0.45 & $\tau_{\mathrm{HCCC}}(10)$ \\
\hline 54 & 446 & & $448 w$ & 4.02 & 0.95 & $\delta_{\mathrm{cco}}(10)$ \\
\hline 55 & 476 & & $469 w$ & 0.41 & 0.41 & $\delta_{\text {cco }}(10)$ \\
\hline 56 & 481 & $481 w$ & & 1.63 & 1.09 & $\tau_{\text {ссос }}(11)+\tau_{\text {носс }}(12)$ \\
\hline 57 & 497 & & & 2.25 & 0.71 & $\delta_{\mathrm{ccC}}(11)$ \\
\hline 58 & 503 & & & 3.69 & 1.57 & $\tau_{\text {носс }}(13)$ \\
\hline 59 & 514 & $511 w$ & & 0.30 & 0.99 & $\delta_{\mathrm{coc}}(14)$ \\
\hline 60 & 517 & & & 3.71 & 0.52 & $\delta_{\mathrm{C} 10 \mathrm{C} 8 \mathrm{C} 3}(10)$ \\
\hline 61 & 523 & $522 w$ & $520 w$ & 3.30 & 3.07 & $v_{\text {сс }}(10)+v_{\text {ос }}(10)+\tau_{\text {носС }}(22)+\tau_{\text {носн }}(10)$ \\
\hline 62 & 541 & & & 1.15 & 1.65 & $\delta_{\mathrm{CCC}}(10)$ \\
\hline 63 & 546 & & & 2.37 & 1.18 & $\delta_{\text {cCC }}(10)$ \\
\hline 64 & 548 & & $563 w$ & 1.26 & 1.93 & $v_{\mathrm{CC}}(10)$ \\
\hline 65 & 576 & $561 w$ & & 0.31 & 0.67 & $\delta_{\text {occ }}(13)$ \\
\hline 66 & 597 & & & 2.97 & 1.77 & $\delta_{\text {occ }}(11)$ \\
\hline 67 & 605 & $608 w$ & $607 w$ & 2.01 & 0.48 & $\tau_{\mathrm{HCCC}}(10)$ \\
\hline 68 & 616 & & & 1.10 & 4.59 & $\tau_{\mathrm{CCCC}}(10)+\tau_{\mathrm{CCCH}}(10)$ \\
\hline 69 & 621 & $626 w$ & & 0.97 & 0.15 & $\tau_{\mathrm{cccc}}(12)+\tau_{\mathrm{occc}}(10)$ \\
\hline 70 & 630 & $637 w$ & & 1.21 & 2.15 & $\delta_{\mathrm{CCC}}(35)$ \\
\hline 71 & 642 & & $643 w$ & 0.81 & 3.90 & $v_{\mathrm{cc}}(16)$ \\
\hline 72 & 661 & & $663 \mathrm{~ms}$ & 0.30 & 0.67 & $\delta_{\text {coc }}(12)$ \\
\hline 73 & 666 & $668 w$ & & 4.51 & 0.24 & $\tau_{\mathrm{ccoc}}(14)$ \\
\hline 74 & 682 & & & 0.94 & 2.08 & $v_{\mathrm{cc}}(21)$ \\
\hline 75 & 700 & $702 w$ & $704 w$ & 7.31 & 0.71 & $\tau_{\mathrm{H} 37-036-\mathrm{C} 33-\mathrm{C} 31}(10)$ \\
\hline 76 & 706 & & & 2.47 & 0.58 & $\tau_{\text {сссC }}(26)$ \\
\hline 77 & 713 & & & 8.57 & 0.83 & $\tau_{\text {носс }}(20)$ \\
\hline 78 & 718 & $730 w$ & & 0.23 & 0.55 & $\tau_{\mathrm{cccc}}(31)+\tau_{\mathrm{occc}}(21)$ \\
\hline 79 & 752 & $743 w$ & $748 w$ & 14.50 & 0.21 & $\left.\tau_{\mathrm{C} 49--048-\mathrm{C} 42-\mathrm{C} 4 \mathrm{C} 1}(14)\right)+\tau_{\mathrm{H} 49-048-\mathrm{C} 42-\mathrm{C} 43}(27)+\tau_{\mathrm{H} 49-048}$ \\
\hline 80 & 774 & & & 5.37 & 0.49 & $\tau_{\text {носс }}(22)+\tau_{\text {носн }}(10)$ \\
\hline 81 & 784 & & & 2.59 & 1.96 & $\tau_{\mathrm{H} 12-\mathrm{C} 10-\mathrm{C} 8 \mathrm{-O} 015}(20)+\tau_{\mathrm{O} 35-\mathrm{C} 11-\mathrm{C} 10-\mathrm{H} 12}(20)$ \\
\hline 82 & 788 & & & 2.02 & 1.41 & $\tau_{\mathrm{H} 25-\mathrm{C} 20-\mathrm{C} 118-\mathrm{C} 17}(20)+\tau_{\mathrm{C} 22-\mathrm{C} 24-\mathrm{CL} 2-\mathrm{H} 25}(14)+\tau_{\text {O27-C24- }}$ \\
\hline 83 & 794 & & & 4.26 & 2.11 & $v_{\mathrm{C} 69-\mathrm{C} 44}(15)$ \\
\hline 84 & 796 & & & 2.84 & 2.84 & $v_{027-C 24}(14)$ \\
\hline 85 & 805 & & & 1.86 & 1.69 & $v_{\mathrm{CC}}(18)+v_{\mathrm{oc}}(11)$ \\
\hline 86 & 812 & & $812 w$ & 6.63 & 0.48 & $\tau_{\text {C24-C22-C19-H23 }}(13)+\tau_{\mathrm{H} 26-\mathrm{C} 22-\mathrm{C} 19-\mathrm{Cl} 17}(17)+\tau_{\text {027-C24- }}$ \\
\hline 87 & 814 & & & 1.23 & 4.67 & $v_{034-C 32}(18)$ \\
\hline
\end{tabular}




\begin{tabular}{|c|c|c|c|c|c|c|}
\hline 88 & 823 & $821 \mathrm{~ms}$ & & 5.97 & 0.24 & $v_{\mathrm{cc}}(17)+v_{\mathrm{oc}}(11)$ \\
\hline 89 & 848 & $835 w$ & $837 w$ & 1.61 & 2.71 & $v_{\mathrm{C} 18-\mathrm{C} 17}(11)$ \\
\hline 90 & 856 & & & 0.95 & 0.28 & $v_{\mathrm{C} 33-\mathrm{C} 31}(14)$ \\
\hline 91 & 871 & $864 \mathrm{vw}$ & $864 w$ & 0.45 & 1.47 & $v_{\mathrm{C} 43-\mathrm{C} 42}(15)$ \\
\hline 92 & 876 & $888 w$ & & 2.61 & 0.89 & $v_{013-C 1}(16)$ \\
\hline 93 & 877 & & & 6.17 & 0.34 & $v_{\mathrm{cc}}(22)+v_{\mathrm{oc}}(23)$ \\
\hline 94 & 910 & & $896 \mathrm{~ms}$ & 0.22 & 0.34 & $\tau_{\mathrm{H} 21-\mathrm{C} 18-\mathrm{C} 17-\mathrm{Cl} 1}(15)+\tau_{\mathrm{C} 24-\mathrm{C} 20-\mathrm{C} 18-\mathrm{H} 21}(11)+\tau_{\mathrm{H} 25-\mathrm{C} 20-}$ \\
\hline 95 & 922 & $919 w$ & & 0.01 & 0.12 & $\begin{array}{l}\tau_{\mathrm{H} 23-\mathrm{C} 19-\mathrm{C} 17-\mathrm{Cl} 1}(12)+\tau_{\mathrm{H} 23-\mathrm{C} 19-\mathrm{C} 17-\mathrm{C} 18}(11)+\tau_{\mathrm{H} 26-\mathrm{C} 22-} \\
\mathrm{C} 19-\mathrm{H} 23\end{array}$ \\
\hline 96 & 923 & & & 1.76 & 2.11 & $v_{046-\mathrm{C} 44}(17)+\delta_{\mathrm{H}-\mathrm{C}-\mathrm{C}}(13)$ \\
\hline 97 & 953 & & & 0.54 & 0.74 & $v_{013-\mathrm{Cl}}(11)+\tau_{\mathrm{HCCC}}(10)$ \\
\hline 98 & 961 & & & 1.34 & 1.10 & $v_{\mathrm{C} 45-\mathrm{C} 43}(24)+\delta_{\mathrm{H} 72-\mathrm{C} 69-\mathrm{C} 44}(11)+\tau_{\mathrm{H} 70-\mathrm{C} 69-\mathrm{C} 44}(10)$ \\
\hline 99 & 963 & & & 2.63 & 0.87 & $v_{\mathrm{C} 31-\mathrm{C} 30}(13)+v_{\text {036-C33 }}(12)$ \\
\hline 100 & 987 & $986 \mathrm{~ms}$ & $982 w$ & 28.45 & 4.40 & $v_{\text {035-C29 }}(20)$ \\
\hline 101 & 990 & & $1002 w$ & 3.25 & 0.12 & $v_{\mathrm{CC}}(31)+\delta_{\mathrm{CCC}}(32)+\delta_{\mathrm{CCH}}(18)+\delta_{\mathrm{HCC}}(14)$ \\
\hline 102 & 1004 & & & 10.29 & 1.40 & $v_{038-c} 31(11)$ \\
\hline 103 & 1011 & & & 38.88 & 0.64 & $v_{\mathrm{oc}}(16)+v_{\mathrm{CC}}(12)$ \\
\hline 104 & 1021 & & & 8.50 & 1.82 & $v_{\mathrm{oc}}(16)+v_{\mathrm{CC}}(10)$ \\
\hline 105 & 1025 & & & 8.95 & 0.44 & $v_{\mathrm{CC}}(11)+v_{\mathrm{CO}}(32)+v_{\mathrm{C} 69-\mathrm{C} 44}(10)$ \\
\hline 106 & 1032 & & & 13.48 & 0.82 & $v_{\mathrm{C} 33-\mathrm{-с32}}(14)$ \\
\hline 107 & 1038 & & & 15.47 & 0.66 & $v_{040-\mathrm{C} 30}(11)+v_{055-\mathrm{C} 54}(24)$ \\
\hline 108 & 1039 & & $1039 w$ & 15.09 & 0.88 & $v_{\mathrm{C5}-\mathrm{C} 1}(14)+v_{\mathrm{C} 33-\mathrm{C} 32}(14)$ \\
\hline 109 & 1040 & $1040 \mathrm{~s}$ & & 3.50 & 3.12 & $v_{\mathrm{C5}-\mathrm{Cl}}(19)+v_{013-\mathrm{Cl} 1}(11)$ \\
\hline 110 & 1053 & & & 57.67 & 0.97 & $v_{C 41-040}(20)+v_{046-C 41}(18)+v_{052-C 45}(18)$ \\
\hline 111 & 1063 & $1060 \mathrm{~s}$ & & 33.94 & 1.22 & $v_{013-\mathrm{C} 2}(12)+v_{015-\mathrm{C} 8}(11)+v_{055-\mathrm{C} 54}(19)$ \\
\hline 112 & 1066 & & & 2.32 & 1.28 & $v_{055-\mathrm{C} 54}(16)+v_{\mathrm{CO}}(35)$ \\
\hline 113 & 1070 & & & 6.68 & 0.27 & $v_{\mathrm{co}}(42)$ \\
\hline 114 & 1073 & & & 7.73 & 0.99 & $v_{\mathrm{co}}(26)$ \\
\hline 115 & 1076 & $1071 \mathrm{~s}$ & & 7.75 & 0.30 & $v_{\text {036-Сз3 }}(19)$ \\
\hline 116 & 1082 & & $1081 \mathrm{~ms}$ & 25.65 & 1.70 & $v_{038-C 31}(15)+v_{C 41-040}(11)+v_{048-C 42}(11)$ \\
\hline 117 & 1087 & $1089 \mathrm{~s}$ & & 1.36 & 0.95 & $v_{\mathrm{C} 22-\mathrm{C} 19}(15)+\delta_{\mathrm{H} 26-\mathrm{C} 22-\mathrm{C} 19}(14)$ \\
\hline 118 & 1095 & & & 15.05 & 0.57 & $v_{\mathrm{co}}(18)$ \\
\hline 119 & 1100 & & & 12.86 & 1.28 & $v_{048-C 42}(29)+v_{052-C 45}(24)$ \\
\hline 120 & 1104 & & & 15.31 & 1.13 & $v_{\mathrm{C} 54-\mathrm{C} 32}(11)$ \\
\hline 121 & 1107 & & & 12.96 & 0.88 & $v_{050-C 43}(12)+v_{052-C 45}(13)$ \\
\hline 122 & 1116 & & $1122 \mathrm{~ms}$ & 8.29 & 0.35 & $\delta_{\mathrm{H} 53-052-\mathrm{C} 45}(11)+\Gamma_{\text {нссн }}(10)$ \\
\hline 123 & 1134 & $1136 s$ & & 17.14 & 0.89 & $v_{046-\mathrm{C} 41}(15)+\delta_{\mathrm{CCH}}(10)$ \\
\hline 124 & 1142 & & & 3.17 & 2.44 & $v_{\mathrm{CO}}(19)+\delta_{\mathrm{CCH}}(14)$ \\
\hline 125 & 1152 & & & 14.20 & 1.00 & $\delta_{\mathrm{H} 21-\mathrm{C} 18-\mathrm{C} 17}(10)+\delta_{\mathrm{H} 26-\mathrm{C} 22-\mathrm{C} 19}(11)+\delta_{\mathrm{C} 24-\mathrm{C} 22-\mathrm{H} 26}(12)$ \\
\hline
\end{tabular}




\begin{tabular}{|c|c|c|c|c|c|c|}
\hline 126 & 1153 & & & 2.97 & 0.92 & $\delta_{\mathrm{C} 24-\mathrm{C} 20-\mathrm{H} 25}(10)+\delta_{\mathrm{H} 28-027-\mathrm{C} 24}(35)$ \\
\hline 127 & 1159 & & & 41.82 & 1.39 & $\delta_{\text {нсс }}(14)+v_{\text {C- }}(23)$ \\
\hline 128 & 1175 & $1178 \mathrm{~s}$ & & 2.39 & 0.59 & $v_{\mathrm{C} 10-\mathrm{C} 8}(11)+\delta_{\mathrm{H} 12-\mathrm{C} 10-\mathrm{C} 8}(13)+\delta_{\mathrm{H} 16-015-\mathrm{C} 8}(36)$ \\
\hline 129 & 1180 & & $1185 w$ & 3.80 & 1.01 & $\delta_{\mathrm{H} 56-055-\mathrm{C} 54}(24)+\delta_{\mathrm{CCH}}(10)+\delta_{\mathrm{H} 37-036-\mathrm{C} 33}(10)$ \\
\hline 130 & 1189 & & & 16.87 & 1.40 & $v_{\mathrm{C} 17 \mathrm{C} 1}(11)$ \\
\hline 131 & 1191 & & & 1.29 & 4.38 & $v_{\mathrm{C} 17-\mathrm{Cl}}(18)$ \\
\hline 132 & 1201 & & & 5.32 & 0.05 & $\delta_{\mathrm{H} 51-050-\mathrm{C} 43}(17)$ \\
\hline 133 & 1203 & $1205 s$ & $1210 \mathrm{~s}$ & 12.61 & 4.78 & $v_{\mathrm{C} 4-\mathrm{C} 3}(10)$ \\
\hline 134 & 1217 & & $1215 w$ & 3.99 & 0.92 & $\delta_{\mathrm{H} 56-055-\mathrm{C} 54}(23)+\delta_{\mathrm{H} 58-\mathrm{C} 54-\mathrm{C} 32}(12)+\delta_{055-\mathrm{C} 54-\mathrm{H} 58}(10)$ \\
\hline 135 & 1224 & & & 2.95 & 1.24 & $\delta_{\mathrm{H} 53-052-\mathrm{C} 45}(31)$ \\
\hline 136 & 1227 & & & 1.00 & 0.77 & $\delta_{\mathrm{H} 68-\mathrm{C5}-\mathrm{Cl}}(16)+\delta_{\mathrm{H} 68-\mathrm{C5}-\mathrm{C} 4}(15)$ \\
\hline 137 & 1245 & & & 0.96 & 0.93 & $\delta_{\mathrm{H} 57-\mathrm{C} 54-\mathrm{C} 32}(13)$ \\
\hline 138 & 1248 & & & 7.72 & 0.73 & $\delta_{\mathrm{H} 49-048-\mathrm{C} 42}(14)$ \\
\hline 139 & 1254 & & $1254 w$ & 41.32 & 6.38 & $v_{\mathrm{C} 4-\mathrm{C3}}(18)+\delta_{\mathrm{H} 16-015-\mathrm{C} 8}(19)$ \\
\hline 140 & 1256 & & & 1.73 & 0.24 & $\delta_{\mathrm{C} 43-\mathrm{C} 42-\mathrm{H} 63}(11)$ \\
\hline 141 & 1259 & & & 8.28 & 2.64 & $v_{027-C 24}(48)$ \\
\hline 142 & 1260 & $1263 w$ & & 0.21 & 0.44 & $\delta_{\text {нос }}(14)$ \\
\hline 143 & 1279 & & & 0.17 & 1.21 & $\delta_{\mathrm{CCH}}(22)$ \\
\hline 144 & 1284 & $1282 w$ & & 5.76 & 0.62 & $v_{\mathrm{C} 18-\mathrm{C} 17}(12)$ \\
\hline 145 & 1297 & $1296 s$ & & 1.93 & 1.15 & $v_{\mathrm{C} 45-\mathrm{C} 44}(11)+\delta_{\text {O46-C44-H60}}(11)$ \\
\hline 146 & 1310 & & & 6.20 & 1.91 & $\delta_{\mathrm{CCH}}(14)+\delta_{\mathrm{OCH}}(10)$ \\
\hline 147 & 1312 & & & 6.75 & 1.57 & $\delta_{\mathrm{CCH}}(13)+\delta_{\mathrm{OCH}}(10)+v_{\mathrm{COCH}}(12)$ \\
\hline 148 & 1315 & & & 5.24 & 0.39 & $\delta_{\mathrm{H} 51-050-\mathrm{C} 43}(23)$ \\
\hline 149 & 1322 & & & 11.27 & 0.37 & $v_{\mathrm{cc}}(15)$ \\
\hline 150 & 1324 & & & 0.67 & 1.01 & $\delta_{\mathrm{H} 28-027-\mathrm{C} 24}(10)$ \\
\hline 151 & 1328 & & & 5.73 & 0.68 & $v_{\mathrm{cC}}(31)$ \\
\hline 152 & 1331 & & & 2.80 & 0.65 & $\delta_{\mathrm{CCH}}(10)$ \\
\hline 153 & 1334 & & & 4.28 & 0.43 & $\delta_{\text {нос }}(10)+\Gamma_{\text {нсСн }}(11)$ \\
\hline 154 & 1338 & & & 0.28 & 0.41 & $\delta_{034-\mathrm{C} 32-\mathrm{H} 66}(12)$ \\
\hline 155 & 1344 & $1341 \mathrm{~ms}$ & & 7.17 & 1.37 & $\delta_{\text {occ }}(10)$ \\
\hline 156 & 1345 & & & 1.95 & 0.85 & $\delta_{\text {нсС }}(25)$ \\
\hline 157 & 1349 & & $1348 \mathrm{~ms}$ & 1.73 & 0.32 & $\delta_{\mathrm{HCC}}(13)+\Gamma_{\mathrm{HCCH}}(11)$ \\
\hline 158 & 1354 & & & 11.74 & 1.66 & $v_{015-c 8}(12)$ \\
\hline 159 & 1357 & & & 3.85 & 0.21 & $\delta_{\text {нос }}(11)$ \\
\hline 160 & 1363 & $1363 \mathrm{~ms}$ & & 1.88 & 0.30 & $\delta_{\mathrm{H} 71-\mathrm{C} 69-\mathrm{H} 72}(20)+\delta_{\mathrm{HCC}}(18)$ \\
\hline 161 & 1370 & & $1373 w$ & 0.57 & 0.51 & $\delta_{\text {034-С29-н67 }}(12)+\Gamma_{\text {н59-с30-с29-н67 }}(11)$ \\
\hline 162 & 1378 & & & 1.81 & 0.62 & $\delta_{\text {нос }}(12)+\delta_{\text {нсо }}(10)$ \\
\hline 163 & 1383 & & & 2.41 & 0.34 & $\delta_{\mathrm{COH}}(11)$ \\
\hline 164 & 1390 & $1393 \mathrm{~ms}$ & & 12.51 & 0.24 & $\delta_{\mathrm{H} 39-038-\mathrm{C3} 1}(33)$ \\
\hline
\end{tabular}




\begin{tabular}{|c|c|c|c|c|c|c|}
\hline 165 & 1399 & & & 2.48 & 0.80 & $\delta_{\mathrm{H} 37-036-\mathrm{C} 33}(26)+\delta_{\mathrm{H} 49-048-\mathrm{C} 42}(14)$ \\
\hline 166 & 1407 & & & 1.85 & 0.15 & $\delta_{\text {н51-050-С43 }}(13)+\delta_{\text {нос }}(18)$ \\
\hline 167 & 1408 & & & 1.25 & 1.84 & $\delta_{\text {н9-С5-н68 }}(31)+\Gamma_{\text {нсСС }}(25)+\Gamma_{\text {нсСо }}(25)+\Gamma_{\text {нсСН }}(10)$ \\
\hline 168 & 1411 & & & 10.03 & 1.15 & $\delta_{\mathrm{H} 37-036-\mathrm{C} 33}(15)+\delta_{\mathrm{H} 39-038-\mathrm{C} 31}(14)+\delta_{\mathrm{H} 49-048-\mathrm{-C42}}(17)$ \\
\hline 169 & 1420 & & & 13.63 & 0.06 & $v_{\mathrm{CC}}(29)+\delta_{\mathrm{CCH}}(11)$ \\
\hline 170 & 1423 & & & 1.69 & 0.28 & $\delta_{\mathrm{H} 56-055-\mathrm{C} 54}(13)+\delta_{\mathrm{H} 57-\mathrm{C} 54-\mathrm{C} 32}(25)+\Gamma_{\text {носС }}(19)$ \\
\hline 171 & 1429 & & & 16.16 & 0.06 & $\delta_{\mathrm{CCC}}(25)+\delta_{\mathrm{CCH}}(11)$ \\
\hline 172 & 1447 & & & 0.60 & 1.85 & $\delta_{\mathrm{H} 70-\mathrm{C} 69-\mathrm{C} 44}(11)+\delta_{\mathrm{H} 70-\mathrm{C} 69-\mathrm{H} 71}(24)+\delta_{\mathrm{H} 71-\mathrm{C} 69-\mathrm{H} 72}(23)$ \\
\hline 173 & 1450 & $1453 \mathrm{~ms}$ & $1456 \mathrm{~ms}$ & 0.08 & 1.04 & $\delta_{\mathrm{H} 70-\mathrm{C} 69-\mathrm{H} 72}(39)+\Gamma_{\mathrm{HCCH}}(10)$ \\
\hline 174 & 1466 & & & 0.99 & 1.30 & $\delta_{\mathrm{H} 57-\mathrm{C} 54-\mathrm{H} 58}(30)+\Gamma_{\mathrm{HCCC}}(16)+\Gamma_{\text {нссо }}(16)+\Gamma_{\text {нсСн }}(17)$ \\
\hline 175 & 1473 & & & 4.50 & 0.18 & $v_{\mathrm{C} 11-\mathrm{C} 7}(17)$ \\
\hline 176 & 1502 & $1502 \mathrm{~ms}$ & $1502 \mathrm{vw}$ & 12.45 & 0.71 & $v_{\mathrm{C} 18-\mathrm{C} 17}(10)$ \\
\hline 177 & 1561 & & $1574 \mathrm{~ms}$ & 20.70 & 0.96 & $v_{\mathrm{C} 3-\mathrm{C} 2}(12)+v_{\mathrm{C} 8-\mathrm{C} 3}(13)+v_{\mathrm{C} 11-\mathrm{C} 7}(12)+v_{\mathrm{C} 11-\mathrm{C} 10}(28)$ \\
\hline 178 & 1584 & $1582 \mathrm{~ms}$ & & 3.29 & 0.61 & $\begin{array}{l}v_{\mathrm{C} 18-\mathrm{C} 17}(11)+v_{\mathrm{C} 19-\mathrm{C} 17}(20)+v_{\mathrm{C} 24-} \\
\mathrm{C} 20(20)+v_{\mathrm{C} 24-\mathrm{C} 22}(17)\end{array}$ \\
\hline 179 & 1598 & & & 105.6 & 14.03 & $v_{\mathrm{C} 7 \cdot \mathrm{C} 2}(22)+v_{\mathrm{C} 10-\mathrm{C} 8}(20)+v_{\mathrm{C} 11-\mathrm{C} 7}(12)$ \\
\hline 180 & 1610 & & & 7.51 & 8.61 & $v_{\mathrm{C} 20-\mathrm{C} 18}(20)+v_{\mathrm{C} 22-\mathrm{C} 19}(20)+v_{\mathrm{C} 24-\mathrm{C} 22}(10)$ \\
\hline 181 & 1710 & $1646 s$ & $1643 v s$ & 36.22 & 7.18 & $v_{014-C 4}(87)$ \\
\hline 182 & 2865 & & & 6.63 & 2.26 & $v_{\mathrm{C} 54-\mathrm{H} 57}(70)+v_{\mathrm{C} 54-\mathrm{H} 58}(30)$ \\
\hline 183 & 2873 & & & 0.65 & 0.73 & $v_{\mathrm{C} 41-\mathrm{H} 47}(20)+v_{\mathrm{C} 45-\mathrm{H} 61}(33)+v_{\mathrm{C} 43-\mathrm{H} 62}(39)$ \\
\hline 184 & 2883 & & & 2.33 & 1.37 & $v_{\mathrm{C} 41-\mathrm{H} 47}(39)+v_{\mathrm{C} 45-\mathrm{H} 61}(11)+v_{\mathrm{C} 43-\mathrm{H} 62}(49)$ \\
\hline 185 & 2888 & $2890 w$ & & 3.24 & 1.62 & $v_{\mathrm{C} 1-\mathrm{H} 73}(97)$ \\
\hline 186 & 2890 & & & 4.81 & 2.82 & $v_{\mathrm{C} 41-\mathrm{H} 47}(29)+v_{\mathrm{C} 45-\mathrm{H} 61}(51)+v_{\mathrm{C} 42-\mathrm{H} 63}(19)$ \\
\hline 187 & 2904 & & $2899 \mathrm{~ms}$ & 4.31 & 1.64 & $v_{\mathrm{C} 54-\mathrm{H} 57}(28)+v_{\mathrm{C} 54-\mathrm{H} 58}(69)$ \\
\hline 188 & 2907 & & & 13.46 & 0.29 & $v_{\mathrm{C} 41-\mathrm{H} 47}(12)+v_{\mathrm{C} 43-\mathrm{H} 62}(12)+v_{\mathrm{C} 42-\mathrm{H} 63}(70)$ \\
\hline 189 & 2927 & $2929 w$ & & 1.27 & 1.28 & $v_{\mathrm{C5}-\mathrm{H} 9}(87)+v_{\mathrm{C5}-\mathrm{H} 68}(11)$ \\
\hline 190 & 2938 & & & 1.52 & 3.52 & $\begin{array}{l}v_{\mathrm{C} 44-\mathrm{H} 60}(13)+v_{\mathrm{C} 69-\mathrm{H} 70}(41)+v_{\mathrm{C} 69-} \\
{ }_{\mathrm{H} 71}(20)+v_{\mathrm{C} 69-\mathrm{H} 72}(25)\end{array}$ \\
\hline 191 & 2939 & & $2939 w$ & 2.77 & 0.78 & $v_{\mathrm{C} 29-\mathrm{H} 67}(97)$ \\
\hline 192 & 2941 & & & 4.72 & 1.18 & $v_{\mathrm{C} 44-\mathrm{H} 60}(83)$ \\
\hline 193 & 2943 & & & 1.93 & 1.77 & $v_{\mathrm{C} 33-\mathrm{H} 65}(23)+v_{\mathrm{C} 32-\mathrm{H} 66}(73)$ \\
\hline 194 & 2952 & & $2958 w$ & 1.95 & 0.98 & $v_{\mathrm{C} 31-\mathrm{H} 64}(23)+v_{\mathrm{C} 33-\mathrm{H} 65}(55)+v_{\mathrm{C} 32-\mathrm{H} 66}(21)$ \\
\hline 195 & 2965 & & & 6.01 & 2.23 & $v_{\mathrm{C} 31-\mathrm{H} 64}(73)+v_{\mathrm{C} 33-\mathrm{H} 65}(22)$ \\
\hline 196 & 2985 & $2970 w$ & 2976w & 2.17 & 1.36 & $v_{\mathrm{C} 30-\mathrm{H} 59}(95)$ \\
\hline 197 & 3005 & & & 1.16 & 2.05 & $v_{\text {с5-H9 }}(10)+v_{\text {C5-H68 }}(89)$ \\
\hline 198 & 3008 & & & 4.42 & 0.75 & $v_{\mathrm{C} 69 \mathrm{H} 70}(52)+v_{\mathrm{C} 69-\mathrm{H} 72}(42)$ \\
\hline 199 & 3021 & & & 1.75 & 1.53 & $v_{\mathrm{C} 69-\mathrm{H} 71}(71)+v_{\mathrm{C} 69-\mathrm{H} 72}(25)$ \\
\hline 200 & 3042 & & & 3.33 & 1.72 & $v_{\mathrm{C} 20-\mathrm{H} 25}(96)$ \\
\hline 201 & 3053 & & & 1.14 & 1.28 & $v_{\mathrm{C} 19-\mathrm{H} 23}(95)$ \\
\hline 202 & 3083 & & $3071 w$ & 0.88 & 0.96 & $v_{\mathrm{C} 18-\mathrm{H} 21}(95)$ \\
\hline
\end{tabular}




\begin{tabular}{|c|c|c|c|c|c|}
\hline 203 & 3089 & & 0.86 & 3.57 & $v_{\mathrm{C} 22-\mathrm{H} 26}(94)$ \\
\hline 204 & 3096 & & 0.89 & 1.70 & $v_{\mathrm{C} 10-\mathrm{H} 12}(100)$ \\
\hline 205 & 3114 & & 0.01 & 1.24 & $v_{\mathrm{C} 7 \mathrm{H} 6}(100)$ \\
\hline 206 & 3425 & $3427 \mathrm{~s}$ & 69.82 & 1.75 & $v_{\text {O48-H49 }}(95)$ \\
\hline 207 & 3497 & & 12.72 & 0.40 & $v_{\text {038-н39 }}(92)$ \\
\hline 208 & 3555 & & 17.68 & 0.40 & $v_{\text {036-н37 }}(97)$ \\
\hline 209 & 3603 & & 8.26 & 0.53 & $v_{050-\mathrm{H} 51}(100)$ \\
\hline 210 & 3653 & & 5.73 & 1.47 & $v_{015-\mathrm{H} 16}(100)$ \\
\hline 211 & 3658 & & 4.70 & 1.07 & $v_{052-\mathrm{H} 53}(100)$ \\
\hline 212 & 3672 & & 7.22 & 1.56 & $v_{\text {027-H28 }}(100)$ \\
\hline 213 & 3680 & & 3.36 & 1.75 & $v_{055-\mathrm{H} 56}(100)$ \\
\hline
\end{tabular}

v: Stretching, $\delta$ : In-plane-bending, $\gamma$ : Out-of-plane bending, vw: Very week, w: Week, m: Medium, s: Strong, vs: Very strong, Scaling factor: 0.9608 (palafox, 2000),

${ }^{\mathrm{b}}$ Relative IR Absorption intensities normalized with highest peak absorption equal to 100 ,

${ }^{\mathrm{c}}$ Relative raman intensities calculated by equation (2.1) and normalized to 100.

d Total energy distribution calculated at B3LYP/6-31G(d,p) level.

harmonic bands 961, $1116 \mathrm{~cm}^{-1}$ (mode numbers: 98, 122) are assigned as rocking modes of $\mathrm{CH}_{3}$. The torsion vibrations are not observed in the FT-IR spectrum because this appears at very low frequency. The FT-Raman experimental band observed at 220 $\mathrm{cm}^{-1}$ shows an excellent agreement with theoretical results. These assignments support from the work of Sundaraganesan et al. and are within the frequency intervals given by Varsanyi $[25,26]$.

\section{Methylene Group $\left(\mathrm{CH}_{2}\right)$ Vibrations}

In present molecule, two methylene groups are available, which are attached in Naringenin core and in D-glucose structure. In which, the methylene group in Naringenin core has observed at higher frequency than in ring D. The symmetric and asymmetric $\left(\mathrm{H}_{68}-\mathrm{C}_{5}-\mathrm{H}_{9}\right)$ stretching modes are assigned to $2929 \mathrm{~cm}^{-1} / \mathrm{FT}-\mathrm{IR}$ (2927/mode number: 189) and $3005 \mathrm{~cm}^{-1}$ (mode number: 197) respectively. Similarly, the harmonic bands $2865 \mathrm{~cm}^{-1}$ (mode number: 182) and 2904 (mode number: 187)/ $2899 \mathrm{~cm}^{-1}$ : Raman are assigned to $\delta \mathrm{CH}_{2}$ (sym) and $\delta \mathrm{CH}_{2}\left(\mathrm{H}_{57}-\mathrm{C}_{54}-\mathrm{H}_{58}\right)$ (asym) mode respectively. These assignments are well comparable and also find support from TED column. The methylene group has six normal modes of vibrations, namely $\mathrm{CH}_{2}$ symmetric (vsym), $\mathrm{CH}_{2}$ asymmetric (vasym), scissoring $(\delta)$, rocking $(\rho)$, wagging $(\omega)$ and twisting $(\mathrm{t})$ modes are able to appear in the range $1500-800 \mathrm{~cm}^{-1}$ [22].

In this molecule, there are two $\mathrm{CH}_{2}$ groups (ring B \& D). The general order of $\mathrm{CH}_{2}$ deformation is: $\mathrm{CH}_{2}$ scissoring $>\mathrm{CH}_{2}$ wagging $>\mathrm{CH}_{2}$ twisting $>\mathrm{CH}_{2}$ rocking. In the present study, the $\mathrm{CH}_{2}$ bending modes follow the same trend. Since the bending modes involving hydrogen atom attached to the central carbon fall into the range 1450-875 $\mathrm{cm}^{-1}$ there are extensive vibrational coupling of these modes with $\mathrm{CH}_{2}$ deformations particularly with the $\mathrm{CH}_{2}$ twist [26]. It is notable that both $\mathrm{CH}_{2}$ scissoring and $\mathrm{CH}_{2}$ rocking were sensitive to the molecular conformation. The mode numbers: 167, 174 are belonging to the $\delta \mathrm{CH}_{2}$ mode. The frequencies observed at $1185 \mathrm{~cm}^{-1}$ (FT-Raman) and at $1136 \mathrm{~cm}^{-1}$ (FT-IR) are assigned to $\mathrm{CH}_{2}$ wagging and $\mathrm{CH}_{2}$ twisting, respectively. The other fundamental mode of $\mathrm{CH}_{2}$ (rocking: mode number: 97) is observed in the expected region and also presented in Table 1. These assignments are found to be satisfactorily in agreement with the reported values [27].

\section{0-H Vibrations}

The broad, intense $-\mathrm{OH}$ stretching absorption from 3300 to $2500 \mathrm{~cm}^{-1}$ suggests the presence of carboxylic group in the Naringin extracted from kinnow peel. The strong and broad hydrogen bonded $\mathrm{O}-\mathrm{H}$ stretching bands centering 3300 and $3400 \mathrm{~cm}^{-1}$ are for alcohols and phenols, respectively [28]. The hydroxyl group vibrations are appeared in the higher range than other vibrations. In our study, the $\mathrm{O}-\mathrm{H}$ group stretching vibration of $\mathrm{A}, \mathrm{B}, \mathrm{C}, \mathrm{D}$ and $\mathrm{E}$ rings are lies at different frequencies. In this molecule, the mode numbers: 213, 208, 207 and 211, 209, 206 are assigned to the $v(\mathrm{OH})$ for ring $\mathrm{D}$ and ring E, respectively. The harmonic values $3653 \mathrm{~cm}^{-1}$ (mode number: $210 /$ ring A) and 3672 $\mathrm{cm}^{-1}$ (mode number: $212 /$ ring $\mathrm{C}$ ) are belong to the core molecule of Naringin. The experimental $\mathrm{vOH}$ group vibration has observed at $3427 \mathrm{~cm}^{-1}$ as a shoulder band in FT-IR spectrum, which is exactly matches with the literature value $3433 \mathrm{~cm}^{-1}$ [29].

The $\mathrm{OH}$ in-plane bending vibration appears in the range of $1440-1395 \mathrm{~cm}^{-1}$. Akkaya and Akyüz et al. assigned this vibration at 1294 and $1160 \mathrm{~cm}^{-1}$ in IR for 4-aminosalicylic acid [18, 30]. For 3 -aminosalicylic acid, this band is observed at 1340 and $1171 \mathrm{~cm}^{-1}$ which is a motion of hydroxyl group [31]. In the present work, the $\mathrm{O}-\mathrm{H}$ in-plane bending mode is assigned to $1393 \mathrm{~cm}^{-1}$ in FT-IR and 1254, 1185, $1122 \mathrm{~cm}^{-1}$ in FT-Raman experimentally, which are calculated at 1390, 1254, 1180, 1116 (mode numbers: 164, 139, $129,122)$ for Naringin. The above assignments are supported by TED values. The $\delta \mathrm{COH}$ and $\tau \mathrm{COH}$ vibrations (harmonic) are calculated in the regions $1423-1153 \mathrm{~cm}^{-1}$ (mode numbers: 170 126) and 774-228 $\mathrm{cm}^{-1}$ (mode numbers: 80-27), respectively. The observed bands 1215 (FT-Raman), $1263 \mathrm{~cm}^{-1}$ (FT-IR) and 
Table 1: The vibrational assignments of Naringin using Scaled Quantum Mechanics method (B3LYP/6-31G(d,p)).

\begin{tabular}{|c|c|c|c|c|c|}
\hline $\begin{array}{l}\text { Scaled } \\
\text { B3LYPa }^{\mathrm{a}}\end{array}$ & FT-IR & $\begin{array}{c}\text { FT- } \\
\text { Raman }\end{array}$ & $I_{I R}^{b}$ & $I_{\text {Raman }}^{c}$ & $\begin{array}{l}\text { Vibrational assignments } \\
\operatorname{TED}^{d}(\geq 10)\end{array}$ \\
\hline 82 & & $86 s$ & 0.37 & 1.09 & $\tau \operatorname{cccc}(16)+\Gamma \operatorname{occc}(13)$ \\
\hline 132 & & $140 w$ & 0.14 & 2.02 & $\tau \operatorname{ccco}(10)$ \\
\hline 169 & & $168 w$ & 0.50 & 1.30 & $\delta \operatorname{ccc}(15)$ \\
\hline 220 & & $220 w$ & 1.31 & 0.59 & $\delta_{\mathrm{C} 45-\mathrm{C} 44-\mathrm{C} 69}(14)+\tau_{\mathrm{HCCC}}(10)$ \\
\hline 240 & & $247 w$ & 8.53 & 3.46 & $\tau_{\mathrm{H} 56-055-\mathrm{C} 54-\mathrm{C} 32}(32)+\tau_{\mathrm{H} 56-055-\mathrm{C} 54-\mathrm{H} 57}(22)+\tau_{\mathrm{H} 56-055-}$ \\
\hline 376 & & $385 w$ & 0.98 & 0.86 & $\delta_{\text {oCC }}(10)$ \\
\hline 435 & $434 w$ & & 5.92 & 0.45 & $\tau_{\mathrm{HCCC}}(10)$ \\
\hline 446 & & $448 w$ & 4.02 & 0.95 & $\delta_{\mathrm{CCO}}(10)$ \\
\hline 476 & & $469 w$ & 0.41 & 0.41 & $\delta_{\mathrm{CCO}}(10)$ \\
\hline 481 & $481 w$ & & 1.63 & 1.09 & $\tau_{\text {Ссос }}(11)+\tau_{\text {носс }}(12)$ \\
\hline 514 & $511 w$ & & 0.30 & 0.99 & $\delta_{\operatorname{coc}}(14)$ \\
\hline 523 & $522 w$ & $520 w$ & 3.30 & 3.07 & $v_{\text {СС }}(10)+v_{\text {ос }}(10)+\tau_{\text {носС }}(22)+\tau_{\text {носн }}(10)$ \\
\hline 548 & & $563 w$ & 1.26 & 1.93 & $v_{\mathrm{CC}}(10)$ \\
\hline 576 & $561 w$ & & 0.31 & 0.67 & $\delta_{\mathrm{OCC}}(13)$ \\
\hline 597 & & & 2.97 & 1.77 & $\delta_{\mathrm{OCC}}(11)$ \\
\hline 605 & $608 w$ & $607 w$ & 2.01 & 0.48 & $\tau_{\mathrm{HCCC}}(10)$ \\
\hline 616 & & & 1.10 & 4.59 & $\tau_{\mathrm{CCCC}}(10)+\tau_{\mathrm{CCCH}}(10)$ \\
\hline 621 & $626 w$ & & 0.97 & 0.15 & $\tau_{\mathrm{CCCC}}(12)+\tau_{\mathrm{OCCC}}(10)$ \\
\hline 642 & & $643 w$ & 0.81 & 3.90 & $v_{\mathrm{CC}}(16)$ \\
\hline 661 & & $663 \mathrm{~ms}$ & 0.30 & 0.67 & $\delta_{\text {Coc }}(12)$ \\
\hline 666 & $668 w$ & & 4.51 & 0.24 & $\tau_{\mathrm{cCoc}}(14)$ \\
\hline 700 & $702 w$ & $704 w$ & 7.31 & 0.71 & $\tau_{\mathrm{H} 37-036-\mathrm{C} 33-\mathrm{C} 31}(10)$ \\
\hline 718 & $730 w$ & & 0.23 & 0.55 & $\tau_{\mathrm{cccc}}(31)+\tau_{\mathrm{occc}}(21)$ \\
\hline 752 & $743 w$ & $748 w$ & 14.50 & 0.21 & $\tau_{\mathrm{H} 49-048-\mathrm{C} 42-\mathrm{C} 41}(30)+\tau_{\mathrm{H} 49-048-\mathrm{C} 42-\mathrm{C} 43}(27)+\tau_{\mathrm{H} 49-048-}$ \\
\hline 812 & & $812 w$ & 6.63 & 0.48 & $\tau_{\mathrm{C} 24-\mathrm{C} 22-\mathrm{C} 19-\mathrm{H} 23}(13)+\tau_{\mathrm{H} 26-\mathrm{C} 22-\mathrm{C} 19-\mathrm{C} 17}(17)+\tau_{\mathrm{O} 27-\mathrm{C} 24-}$ \\
\hline 823 & $821 \mathrm{~ms}$ & & 5.97 & 0.24 & $v_{\mathrm{CC}}(17)+v_{\mathrm{OC}}(11)$ \\
\hline 848 & $835 w$ & $837 w$ & 1.61 & 2.71 & $v_{\mathrm{C} 18-\mathrm{C} 17}(11)$ \\
\hline 871 & $864 \mathrm{vw}$ & $864 w$ & 0.45 & 1.47 & $v_{\mathrm{C} 43-\mathrm{C} 42}(15)$ \\
\hline 876 & $888 w$ & & 2.61 & 0.89 & $v_{013-\mathrm{C} 1}(16)$ \\
\hline 910 & & $896 \mathrm{~ms}$ & 0.22 & 0.34 & $\tau_{\mathrm{H} 21-\mathrm{C} 18-\mathrm{C} 17-\mathrm{C} 1}(15)+\tau_{\mathrm{C} 24-\mathrm{C} 20-\mathrm{C} 18-\mathrm{H} 21}(11)+\tau_{\mathrm{H} 25-\mathrm{C} 20-}$ \\
\hline 922 & $919 w$ & & 0.01 & 0.12 & $\tau_{\mathrm{H} 23-\mathrm{C} 19-\mathrm{C} 17-\mathrm{C} 1}(12)+\tau_{\mathrm{H} 23-\mathrm{C} 19-\mathrm{C} 17-\mathrm{C} 18}(11)+\tau_{\mathrm{H} 26-\mathrm{C} 22-}$ \\
\hline 987 & $986 \mathrm{~ms}$ & $982 w$ & 28.45 & 4.40 & $v_{\text {O35-C29 }}(20)$ \\
\hline 990 & & $1002 w$ & 3.25 & 0.12 & $v_{\mathrm{CC}}(31)+\delta_{\mathrm{CCC}}(32)+\delta_{\mathrm{CCH}}(18)+\delta_{\mathrm{HCC}}(14)$ \\
\hline 1039 & & $1039 w$ & 15.09 & 0.88 & $v_{\mathrm{C} 5-\mathrm{C} 1}(14)+v_{\mathrm{C} 33-\mathrm{C} 32}(14)$ \\
\hline
\end{tabular}




\begin{tabular}{|c|c|c|c|c|c|}
\hline 1040 & $1040 \mathrm{~s}$ & & 3.50 & 3.12 & $v_{\mathrm{C5}-\mathrm{Cl} 1}(19)+v_{013-\mathrm{C} 1}(11)$ \\
\hline 1063 & $1060 \mathrm{~s}$ & & 33.94 & 1.22 & $v_{013-\mathrm{C} 2}(12)+v_{015-\mathrm{Cl}}(11)+v_{055-\mathrm{C} 54}(19)$ \\
\hline 1076 & $1071 \mathrm{~s}$ & & 7.75 & 0.30 & $v_{\text {036-сз3 }}(19)$ \\
\hline 1082 & & $1081 \mathrm{~ms}$ & 25.65 & 1.70 & $v_{038-C 31}(15)+v_{\text {C41-040 }}(11)+v_{048-\mathrm{C} 42}(11)$ \\
\hline 1087 & $1089 \mathrm{~s}$ & & 1.36 & 0.95 & $v_{\mathrm{C} 22-\mathrm{C} 19}(15)+\delta_{\mathrm{H} 26-\mathrm{C} 22-\mathrm{C} 19}(14)$ \\
\hline 1116 & & $1122 \mathrm{~ms}$ & 8.29 & 0.35 & $\delta_{\mathrm{H} 53-052-\mathrm{C4} 5}(11)+\Gamma_{\text {нссн }}(10)$ \\
\hline 1134 & $1136 s$ & & 17.14 & 0.89 & $v_{046-\mathrm{C} 41}(15)+\delta_{\mathrm{CCH}}(10)$ \\
\hline 1175 & $1178 \mathrm{~s}$ & & 2.39 & 0.59 & $v_{\mathrm{C} 10-\mathrm{C} 8}(11)+\delta_{\mathrm{H} 12-\mathrm{C} 10-\mathrm{C} 8}(13)+\delta_{\mathrm{H} 16-015-\mathrm{C} 8}(36)$ \\
\hline 1180 & & $1185 w$ & 3.80 & 1.01 & $\delta_{\mathrm{H} 56-055-\mathrm{C} 54}(24)+\delta_{\mathrm{CCH}}(10)+\delta_{\mathrm{H} 37-036-\mathrm{C3} 3}(10)$ \\
\hline 1203 & $1205 s$ & $1210 s$ & 12.61 & 4.78 & $v_{\mathrm{C} 4-\mathrm{C} 3}(10)$ \\
\hline 1217 & & $1215 w$ & 3.99 & 0.92 & $\delta_{\mathrm{H} 56-055-\mathrm{C} 54}(23)+\delta_{\mathrm{H} 58-\mathrm{C} 54-\mathrm{C} 32}(12)+\delta_{055-\mathrm{C} 54-\mathrm{H} 58}(10)$ \\
\hline 1254 & & $1254 w$ & 41.32 & 6.38 & $v_{\mathrm{C} 4-\mathrm{C3}}(18)+\delta_{\mathrm{H} 16-015-\mathrm{C} 8}(19)$ \\
\hline 1260 & $1263 w$ & & 0.21 & 0.44 & $\delta_{\text {нос }}(14)$ \\
\hline 1284 & $1282 w$ & & 5.76 & 0.62 & $v_{\mathrm{C} 18-\mathrm{C} 17}(12)$ \\
\hline 1297 & $1296 s$ & & 1.93 & 1.15 & $v_{\mathrm{C} 45-\mathrm{C} 44}(11)+\delta_{\text {046-C44-H60 }}(11)$ \\
\hline 1344 & $1341 \mathrm{~ms}$ & & 7.17 & 1.37 & $\delta_{\text {ocC }}(10)$ \\
\hline 1349 & & $1348 \mathrm{~ms}$ & 1.73 & 0.32 & $\delta_{\text {нсС }}(13)+\Gamma_{\text {нссн }}(11)$ \\
\hline 1363 & $1363 \mathrm{~ms}$ & & 1.88 & 0.30 & $\delta_{\mathrm{H} 71-\mathrm{C} 69-\mathrm{H} 72}(20)+\delta_{\mathrm{HCC}}(18)$ \\
\hline 1370 & & $1373 w$ & 0.57 & 0.51 & $\delta_{\text {034-C29-H67 }}(12)+\Gamma_{\mathrm{H} 59-\mathrm{C} 30-\mathrm{C} 29-\mathrm{H} 67}(11)$ \\
\hline 1390 & $1393 \mathrm{~ms}$ & & 12.51 & 0.24 & $\delta_{\text {H39-038-С31 }}(33)$ \\
\hline 1450 & $1453 \mathrm{~ms}$ & $1456 \mathrm{~ms}$ & 0.08 & 1.04 & $\delta_{\mathrm{H} 70-\mathrm{C} 69-\mathrm{H} 72}(39)+\Gamma_{\mathrm{HCCH}}(10)$ \\
\hline 1502 & $1502 \mathrm{~ms}$ & $1502 \mathrm{vw}$ & 12.45 & 0.71 & $v_{\mathrm{C} 18-\mathrm{C} 17}(10)$ \\
\hline 1561 & & $1574 \mathrm{~ms}$ & 20.70 & 0.96 & $v_{\mathrm{C} 3-\mathrm{C} 2}(12)+v_{\mathrm{CB}-\mathrm{C} 3}(13)+v_{\mathrm{C} 11-\mathrm{C} 7}(12)+v_{\mathrm{C} 11-\mathrm{C} 10}(28)$ \\
\hline 1584 & $1582 \mathrm{~ms}$ & & 3.29 & 0.61 & $\begin{array}{l}v_{\mathrm{C} 18-\mathrm{C} 17}(11)+v_{\mathrm{C} 19-\mathrm{C} 17}(20)+v_{\mathrm{C} 24-} \\
{ }_{\mathrm{C} 20}(20)+v_{\mathrm{C} 24-\mathrm{C} 22}(17)\end{array}$ \\
\hline 1710 & $1646 s$ & 1643vs & 36.22 & 7.18 & $v_{014-C 4}(87)$ \\
\hline 2888 & $2890 w$ & & 3.24 & 1.62 & $v_{\mathrm{C} 1-\mathrm{H} 73}(97)$ \\
\hline 2904 & & $2899 \mathrm{~ms}$ & 4.31 & 1.64 & $v_{\mathrm{C} 54-\mathrm{H} 57}(28)+v_{\mathrm{C} 54-\mathrm{H} 58}(69)$ \\
\hline 2927 & $2929 w$ & & 1.27 & 1.28 & $v_{\text {C5-H9 }}(87)+v_{\text {C5-H68 }}(11)$ \\
\hline 2939 & & $2939 w$ & 2.77 & 0.78 & $v_{\mathrm{C} 29-\mathrm{H} 67}(97)$ \\
\hline 2952 & & $2958 w$ & 1.95 & 0.98 & $v_{\mathrm{C} 31-\mathrm{H} 64}(23)+v_{\mathrm{C} 33-\mathrm{H} 65}(55)+v_{\mathrm{C} 32-\mathrm{H} 66}(21)$ \\
\hline 2985 & $2970 w$ & $2976 w$ & 2.17 & 1.36 & $v_{\mathrm{C} 30-\mathrm{H} 59}(95)$ \\
\hline 3083 & & $3071 w$ & 0.88 & 0.96 & $v_{\text {С18-н21 }}(95)$ \\
\hline 3425 & $3427 \mathrm{~s}$ & & 69.82 & 1.75 & $v_{048-\mathrm{H} 49}(95)$ \\
\hline \multicolumn{6}{|c|}{$\begin{array}{l}\text { v: Stretching, } \delta \text { : In-plane-bending, } \tau \text { : Out-of-plane bending, vw: very weak, m: medium, s: Strc } \\
\text { aScaled computed wavenumber using scale factor: } 0.9608 \text { (palafox, 2000), } \\
\text { b Relative IR Absorption intensities normalized with highest peak absorption equal to } 100 \text {, } \\
\text { c Relative raman intensities calculated by equation }(2.1) \text { and normalized to } 100 \text {. } \\
\text { d Total energy distribution calculated at B3LYP/6-31G(d,p) level. }\end{array}$} \\
\hline
\end{tabular}


743/748 $\mathrm{cm}^{-1}$ (FT-IR/FT-Raman), $247 \mathrm{~cm}^{-1}$ : FT-Raman supports the above assignments.

\section{$\mathrm{C}=\mathbf{0}$ and $\mathrm{C}-\mathrm{O}$ Vibrations}

Normal esters are characterized by strong IR absorption due to the carbonyl $(\mathrm{C}=0)$ stretching vibration in the range 1750-1735 $\mathrm{cm}^{-1}$ [32]. The FT-IR and FT-Raman spectra show the vibrational frequency for $\mathrm{C}=0$ at $1646 \mathrm{~cm}^{-1}$ and $1633 \mathrm{~cm}^{-1}$ respectively $[33,29]$. The presence of carbonyl group on Naringin can be confirmed from peak situated at $1634 \mathrm{~cm}^{-1}$ [34]. The carbonyl stretching vibration observed as an intense band at $1603 \mathrm{~cm}^{-1}$ for 6-aminoflavone in FT-IR. The observed wavenumber of the carbonyl stretching vibration is lower due to $\pi$-electron being localized [22]. In the present study the $\mathrm{C}_{4}=\mathrm{O}_{14}$ stretching vibrations is appeared at 1646 (strong) and $1643 \mathrm{~cm}^{-1}$ (very strong) band in FT-IR and FT-Raman spectra respectively, while the harmonic value is $1710 \mathrm{~cm}^{-1}$ (mode number: 181).

In 2, 6-dichloro-4-nitrophenol, the $v_{\mathrm{c}-0}$ vibrations lie in the region $1095-1310 \mathrm{~cm}^{-1}$ [35]. In our study, the $v_{\mathrm{C}-0}$ vibrations are observed at different frequencies in different rings (A, B, C, D \& E). The computed wavenumbers in the range 1354-1053 $\mathrm{cm}^{-1}$ (mode numbers: 158, 141, 124, 123, 121, 119, 118, 116-110) are assigned to $v_{\mathrm{C}-\mathrm{o}}$ mode. These vibrations have considerable TED and also find support from 1136, 1071, $1060 \mathrm{~cm}^{-1}$ (FT-IR) and $1081 \mathrm{~cm}^{-1}$ (FT-Raman). In ring $\mathrm{B}$, the $\mathrm{vO}_{13}-\mathrm{C}_{2}$ is observed at higher frequency (1060 $\mathrm{cm}^{-1}$ : FT-IR/1063: mode number: 111) than the $v \mathrm{O}_{13}-\mathrm{C}_{1}\left(1040 \mathrm{~cm}^{-1}\right.$ : FT-IR/1040: mode number: 109). The harmonic values $\mathrm{C}_{41}-\mathrm{O}_{46}: 1053 \mathrm{~cm}^{-1} /$ mode number: 110 and $\mathrm{C}_{32}-\mathrm{O}_{34}: 814 \mathrm{~cm}^{-1} /$ mode number: 87 are belongs to ring $\mathrm{E}$ and ring $\mathrm{D}$, respectively.

Similarly the computed values $1066 \mathrm{~cm}^{-1} /$ mode number: 112 , $1038 \mathrm{~cm}^{-1} /$ mode number: 107 and $987 \mathrm{~cm}^{-1} /$ mode number: 100 are belongs to $\mathrm{C}_{54}-\mathrm{O}_{55}, \mathrm{C}_{30}-\mathrm{O}_{40}$ and $\mathrm{C}_{29}-\mathrm{O}_{35}$ stretching vibrations, respectively.

\section{C-C Vibrations}

The ring C-C stretching occurs in the region 1625-1430 $\mathrm{cm}^{-}$ 1. The six member aromatic ring has two or three strong bands in the region about $1500 \mathrm{~cm}^{-1}$ are being due to skeletal vibration [33]. The C-C stretching bands for aromatic ring usually appear between 1600 and $1450 \mathrm{~cm}^{-1}$. The $\mathrm{C}$-C stretching of alkenes appeared at $1650 \mathrm{~cm}^{-1}[36,29]$. In the present study, the FT-IR bands at 1582, 1502 (in ring C), and FT-Raman bands at 1574 (ring A), $1502 \mathrm{~cm}^{-1}$ (in ring C) are assigned to $v_{C-C}$ mode. The calculated $v_{C-C}$ frequencies are in the range 1610-1473 $\mathrm{cm}^{-1}$ using B3LYP/6$31 \mathrm{G}(\mathrm{d}, \mathrm{p})$ level. In which the mode numbers: 179, 177, 175 and $180,178,176$ are belongs to ring A and ring C, respectively. The harmonic vibrations 1297 (ring E), 1284 (ring C), 1254 (ring B), 1203 (ring B), 1175 (ring A), 1087 (ring C), 1040 (ring B), 1039 (ring D), $1032 \mathrm{~cm}^{-1}$ (ring D) are also attributed to $v_{C-C}$ mode. These assignments are supported by 1296, 1282, 1205, 1178, 1089, $1040 \mathrm{~cm}^{-1}$ (FT-IR) and 1254, $12101039 \mathrm{~cm}^{-1}$ (FT-Raman) observed bands and also have considerable TED values. The $v_{\mathrm{C}-\mathrm{C}}$ vibrations (other than ring) $1191 \mathrm{~cm}^{-1} /$ mode no: $131,1104 \mathrm{~cm}^{-1}$ / mode no: 120 and $1025 \mathrm{~cm}^{-1} /$ mode no: 105 are belongs to $C_{17}-C_{1}$,
$\mathrm{C}_{54}-\mathrm{C}_{32}$ and $\mathrm{C}_{69}-\mathrm{C}_{44}$, respectively.

In-plane bending deformation $\delta_{\mathrm{HCC}}$ and $\delta_{\mathrm{CCH}}$ are observed at 1348 (FT-Raman), 1215 (FT-Raman), 1178 (FT-IR), 1089 (FTIR) and $1002 \mathrm{~cm}^{-1}$ (FT-Raman) respectively. The observed FT-IR bands: 919, 608, $434 \mathrm{~cm}^{-1} /$ Raman: $896,812,607 \mathrm{~cm}^{-1}$ and FTRaman bands: $896,812 \mathrm{~cm}^{-1}$ are assigned to $\tau_{\mathrm{HCC}}$ and $\tau_{\mathrm{CCH}}$ modes, respectively. Erdoğdu et al., observed the $\delta_{\mathrm{CCH}}$ mode in the region $1495-1001 \mathrm{~cm}^{-1}$ and $\tau_{\text {СCH }}$ mode in the region (FT-IR) 923-451 $\mathrm{cm}^{-1}$ for 6,8-dichloroflavone [33]. The ring breathing mode was calculated at $990 \mathrm{~cm}^{-1} /$ mode number: 101 for Naringin and was observed at $1002 \mathrm{~cm}^{-1}$ in Raman spectra. These assignments are in line with literature [33].

\section{NBO Analysis}

The hyperconjugation may be given as stabilizing effect that arises from an overlap between an occupied orbital with another neighboring electron deficient orbital, when these orbitals are properly orientated. This non-covalent bonding-antibonding interaction can be quantitatively described in terms of the NBO analysis, which is expressed by means of the second-order perturbation interaction energy (E(2)) [37-40][46-49]. This energy represents the estimate of the off-diagonal NBO Fock matrix elements. It can be deduced from the second-order perturbation approach [41]

$$
E^{2}=\Delta E_{i j}=q_{i} \frac{F(i, j)^{2}}{\varepsilon_{j}-\varepsilon_{i}}
$$

where $\mathrm{q}_{\mathrm{i}}$ is the donor orbital occupancy, $\varepsilon_{\mathrm{i}}$ and $\varepsilon_{\mathrm{j}}$ are diagonal elements (orbital energies) and F (i, j) is the off diagonal NBO Fock matrix elements.

The NBO analysis has been carried out to elucidate the intra-molecular interaction among natural bond orbitals, the results of NBO analysis are presented in Table 2. In the present investigation, the $\pi-\pi^{*}$ interaction are mainly interested. Here the electron densities of conjugated $\pi$ bonds are lower than the $\sigma$ bond. Whereas, the delocalization is more while the transition from $\pi$ bond to $\pi^{*}$ bond. The larger $\mathrm{E}^{(2)}$ value, denotes that the more delocalization takes place into a particular bond, it mainly occurs during $\pi-\pi^{*}$ transition. Moreover, as mentioned above the electron densities in donor (i) $\pi$ bonds decreases, at the same time electron density increases in acceptor (j) $\pi^{*}$ bonds. It is evident from the Table 2 the donor ( $\pi$ ) bonds have 1.678, 1.621, 1.667, 1.702 and $1.652 \mathrm{e}$ as electron densities for $\mathrm{C}_{2}-\mathrm{C}_{7}, \mathrm{C}_{3}-\mathrm{C}_{8}, \mathrm{C}_{17}-\mathrm{C}_{18}$, $\mathrm{C}_{19}-\mathrm{C}_{22} \& \mathrm{C}_{20}-\mathrm{C}_{24}$ bond, respectively. These electron densities are relatively lesser than the $\sigma$ bonds. On the other hand, the strong delocalization occurs between donor $\left(\pi: \mathrm{C}_{2}-\mathrm{C}_{7}, \mathrm{C}_{3}-\mathrm{C}_{8}, \mathrm{C}_{17}-\mathrm{C}_{18}\right.$ $\mathrm{C}_{19}-\mathrm{C}_{22}$ and $\mathrm{C}_{20}-\mathrm{C}_{24}$ bonds) and acceptor ( $\pi^{*}: \mathrm{C}_{3}-\mathrm{C}_{8}, \mathrm{C}_{2}-\mathrm{C}_{7}, \mathrm{C}_{19}-\mathrm{C}_{22}$, $\mathrm{C}_{17}-\mathrm{C}_{18}$ and $\mathrm{C}_{17}-\mathrm{C}_{18}$ bonds) bonds, and the electron densities relatively increased in acceptor bonds and hence leading to more stabilization energies are obtained as $51.46,126.44,96.23,75.02$ and $94.73 \mathrm{~kJ} / \mathrm{mol}$, respectively. The NBO analysis explores the insights of intra molecular interactions among the intra bonds in Naringin molecule. 
Table 2: Second order perturbation theory analysis of Fock matrix in NBO basis for Naringin using B3LYP/6-31G(d,p) basis set.

\begin{tabular}{|c|c|c|c|c|c|}
\hline Type & Donor (i) & ED/e & Acceptor (j) & ED/e & $\begin{array}{c}\mathrm{E}^{(2)} \\
\mathrm{kJ} / \mathrm{mol}^{\mathrm{a}}\end{array}$ \\
\hline \multirow[t]{2}{*}{$\pi-\pi^{*}$} & $\mathrm{C}_{2}-\mathrm{C}_{7}(2)$ & 1.678 & $\mathrm{C}_{3}-\mathrm{C}_{8}(2)$ & 0.468 & 51.46 \\
\hline & & & $\mathrm{C}_{10}-\mathrm{C}_{11}(2)$ & 0.429 & 121.92 \\
\hline \multirow[t]{3}{*}{$\pi-\pi^{*}$} & $\mathrm{C}_{3}-\mathrm{C}_{8}(2)$ & 1.621 & $\mathrm{C}_{2}-\mathrm{C}_{7}(2)$ & 0.387 & 126.44 \\
\hline & & & $\mathrm{C}_{4}-\mathrm{O}_{14}(2)$ & 0.159 & 92.05 \\
\hline & & & $\mathrm{C}_{10}-\mathrm{C}_{11}(2)$ & 0.429 & 54.56 \\
\hline \multirow[t]{2}{*}{$\pi-\pi^{*}$} & $C_{10}-C_{11}(2)$ & 1.973 & $\mathrm{C}_{2}-\mathrm{C}_{7}(2)$ & 0.387 & 52.51 \\
\hline & & & $\mathrm{C}_{3}-\mathrm{C}_{8}(2)$ & 0.468 & 122.47 \\
\hline \multirow[t]{2}{*}{$\pi-\pi^{*}$} & $\mathrm{C}_{17}-\mathrm{C}_{18}(2)$ & 1.667 & $\mathrm{C}_{19}-\mathrm{C}_{22}(2)$ & 0.335 & 96.23 \\
\hline & & & $\mathrm{C}_{20}-\mathrm{C}_{24}(2)$ & 0.394 & 75.56 \\
\hline \multirow[t]{2}{*}{$\pi-\pi^{*}$} & $\mathrm{C}_{19}-\mathrm{C}_{22}(2)$ & 1.702 & $\mathrm{C}_{17}-\mathrm{C}_{18}(2)$ & 0.361 & 75.02 \\
\hline & & & $\mathrm{C}_{20}-\mathrm{C}_{24}(2)$ & 0.394 & 95.60 \\
\hline \multirow[t]{2}{*}{$\pi-\pi^{*}$} & $\mathrm{C}_{20}-\mathrm{C}_{24}(2)$ & 1.652 & $\mathrm{C}_{17}-\mathrm{C}_{18}(2)$ & 0.361 & 94.73 \\
\hline & & & $\mathrm{C}_{19}-\mathrm{C}_{22}(2)$ & 0.335 & 71.96 \\
\hline$n-\pi^{*}$ & LP013(2) & 1.837 & $\mathrm{C}_{2}-\mathrm{C}_{7}(2)$ & 0.387 & 121.84 \\
\hline \multirow[t]{2}{*}{$\mathrm{n}-\sigma^{*}$} & LP014(2) & 1.877 & $\mathrm{C}_{3}-\mathrm{C}_{4}$ & 0.071 & 100.17 \\
\hline & & & $\mathrm{C}_{4}-\mathrm{C}_{5}$ & 0.064 & 95.90 \\
\hline $\mathrm{n}-\pi^{*}$ & LP015(2) & 1.838 & $\mathrm{C}_{3}-\mathrm{C}_{8}(2)$ & 0.468 & 152.30 \\
\hline$n-\pi^{*}$ & LPO27(2) & 1.869 & $\mathrm{C}_{20}-\mathrm{C}_{24}(2)$ & 0.394 & 127.32 \\
\hline$n-\pi^{*}$ & LPO34(2) & 1.895 & $\mathrm{C}_{29}-\mathrm{O}_{35}$ & 0.064 & 68.87 \\
\hline \multirow[t]{3}{*}{$\mathrm{n}-\pi^{*}$} & LPO35(2) & 1.827 & $\mathrm{C}_{10}-\mathrm{C}_{11}(2)$ & 0.429 & 121.17 \\
\hline & & & $\mathrm{C}_{29}-\mathrm{O}_{34}$ & 0.059 & 54.10 \\
\hline & & & $\mathrm{C}_{29}-\mathrm{H}_{67}$ & 0.031 & 18.16 \\
\hline \multirow[t]{3}{*}{$\mathrm{n}-\sigma^{*}$} & LPO36(2) & 1.930 & $\mathrm{C}_{31}-\mathrm{C}_{33}$ & 0.051 & 28.58 \\
\hline & & & $\mathrm{C}_{32}-\mathrm{C}_{33}$ & 0.046 & 26.07 \\
\hline & & & $\mathrm{O}_{38}-\mathrm{H}_{39}$ & 0.025 & 18.16 \\
\hline $\mathrm{n}-\sigma^{*}$ & LPO38 & 1.972 & $\mathrm{C}_{31}-\mathrm{C}_{33}$ & 0.051 & 12.30 \\
\hline \multirow[t]{3}{*}{$\mathrm{n}-\sigma^{*}$} & LPO38(2) & 1.937 & $\mathrm{C}_{30}-\mathrm{C}_{31}$ & 0.044 & 26.57 \\
\hline & & & $\mathrm{C}_{31}-\mathrm{H}_{64}$ & 0.026 & 10.96 \\
\hline & & & $\mathrm{O}_{48}-\mathrm{H}_{49}$ & 0.029 & 32.13 \\
\hline \multirow[t]{4}{*}{$n-\sigma^{*}$} & LPO40(1) & 1.949 & $\mathrm{C}_{30}-\mathrm{H}_{59}$ & 0.025 & 13.18 \\
\hline & & & $\mathrm{O}_{36}-\mathrm{H}_{37}$ & 0.019 & 12.59 \\
\hline & & & $\mathrm{C}_{41}-\mathrm{O}_{46}$ & 0.041 & 11.25 \\
\hline & & & $\mathrm{C}_{41}-\mathrm{H}_{47}$ & 0.044 & 13.93 \\
\hline \multirow[t]{3}{*}{$\mathrm{n}-\sigma^{*}$} & LPO40(2) & 1.913 & $\mathrm{C}_{29}-\mathrm{C}_{30}$ & 0.059 & 35.56 \\
\hline & & & $\mathrm{C}_{41}-\mathrm{C}_{42}$ & 0.064 & 28.70 \\
\hline & & & $\mathrm{C}_{41}-\mathrm{O}_{46}$ & 0.041 & 18.45 \\
\hline \multirow[t]{2}{*}{$\mathrm{n}-\sigma^{*}$} & LPO46 & 1.949 & $\mathrm{O}_{40}-\mathrm{C}_{41}$ & 0.048 & 15.82 \\
\hline & & & $\mathrm{C}_{41}-\mathrm{C}_{42}$ & 0.064 & 16.48 \\
\hline
\end{tabular}




\begin{tabular}{|c|c|c|c|c|c|}
\hline & & & $\mathrm{C}_{44}-\mathrm{C}_{45}$ & 0.038 & 14.77 \\
\hline \multirow[t]{5}{*}{$\mathrm{n}-\sigma^{*}$} & LPO46(2) & 1.914 & $\mathrm{O}_{40}-\mathrm{C}_{41}$ & 0.048 & 14.39 \\
\hline & & & $\mathrm{C}_{41}-\mathrm{C}_{42}$ & 0.064 & 11.51 \\
\hline & & & $\mathrm{C}_{41}-\mathrm{H}_{47}$ & 0.044 & 36.36 \\
\hline & & & $\mathrm{C}_{44}-\mathrm{H}_{60}$ & 0.025 & 10.50 \\
\hline & & & $\mathrm{C}_{44}-\mathrm{C}_{69}$ & 0.026 & 24.81 \\
\hline \multirow[t]{2}{*}{$\mathrm{n}-\sigma^{*}$} & LPO48 & 1.970 & $\mathrm{C}_{42}-\mathrm{H}_{63}$ & 0.030 & 13.89 \\
\hline & & & $\mathrm{O}_{50}-\mathrm{H}_{51}$ & 0.016 & 11.59 \\
\hline \multirow[t]{2}{*}{$\mathrm{n}-\sigma^{*}$} & LPO48(2) & 1.947 & $\mathrm{C}_{41}-\mathrm{C}_{42}$ & 0.064 & 39.83 \\
\hline & & & $\mathrm{C}_{42}-\mathrm{H}_{63}$ & 0.030 & 12.72 \\
\hline $\mathrm{n}-\sigma^{*}$ & LPO50 & 1.974 & $\mathrm{C}_{42}-\mathrm{C}_{43}$ & 0.048 & 8.74 \\
\hline \multirow[t]{6}{*}{$\mathrm{n}-\sigma^{*}$} & LPO50(2) & 1.952 & $\mathrm{C}_{42}-\mathrm{C}_{43}$ & 0.048 & 24.06 \\
\hline & & & $\mathrm{C}_{43}-\mathrm{H}_{62}$ & 0.034 & 30.88 \\
\hline & & & $\mathrm{C}_{43}-\mathrm{C}_{45}$ & 0.046 & 32.51 \\
\hline & & & $\mathrm{C}_{45}-\mathrm{H}_{61}$ & 0.032 & 24.23 \\
\hline & & & $\mathrm{C}_{54}-\mathrm{H}_{57}$ & 0.030 & 29.66 \\
\hline & & & $\mathrm{C}_{54}-\mathrm{H}_{58}$ & 0.026 & 27.07 \\
\hline$\pi^{*}-\pi^{*}$ & $\mathrm{C} 3-\mathrm{C} 8 *(2)$ & 0.468 & $\mathrm{C}-\mathrm{O}_{14}{ }^{*}(2)$ & 0.160 & 481.33 \\
\hline$\pi^{*}-\pi^{*}$ & $\mathrm{C} 20-\mathrm{C} 24^{*}(2)$ & 0.394 & $\mathrm{C}_{17}-\mathrm{C}_{18} *(2)$ & 0.361 & 1107.92 \\
\hline
\end{tabular}

\section{Hyperpolarizability calculations}

The first hyperpolarizabilities $(\beta 0)$ polarizability $(\alpha 0)$ and dipole moment $(\mu)$ of Naringin molecule has calculated by B3LYP level of theory using 6-31G $(\mathrm{d}, \mathrm{p})$ basis set, based on the finite-field approach. In the presence of an applied electric field, the energy of a system is a function of the electric field. First hyperpolarizability is a third rank tensor that can be described by a $3 \times 3 \times 3$ matrix. The 27 components of the $3 \mathrm{D}$ matrix can be reduced to 10 components due to Kleinman symmetry [42]. It can be given in the lower tetrahedral format. It is obvious that the lower part of the $3 \times 3 \times 3$ matrix is a tetrahedral. The components of $\beta$ are defined as the coefficients in the Taylor series expansion of the energy in the external electric field. When the external electric field is weak and homogeneous, this expansion becomes:

$$
E=E^{0}-\mu_{\alpha} F_{\alpha}-1 / 2 \alpha_{\alpha \beta} F_{\alpha} F_{\beta}-1 / 6 \beta_{\alpha \beta \gamma} F_{\alpha} F_{\beta} F_{\gamma}
$$

where $\mathrm{E}^{0}$ is the energy of the unperturbed molecules, $\mathrm{F}_{\alpha}$ is the field at the origin, and $\mu_{\alpha}, \alpha_{\alpha \beta}, \beta_{\alpha \beta \gamma}$ is the components of the dipole moment, polarizability and the first hyperpolarizability, respectively. The total static dipole moment $\mu$, the mean polarizability $\alpha_{0}$, the anisotropy of polarizability $\Delta \alpha$ and the mean first hyperpolarizability $\beta_{0}$, using the $\mathrm{x}, \mathrm{y}, \mathrm{z}$ components are defined as

$$
\begin{aligned}
& \mu=\left(\mu_{x}^{2}+\mu_{y}^{2}+\mu_{z}^{2}\right)^{1 / 2} \\
& \alpha_{0}=\frac{\alpha_{x x}+\alpha_{y y}+\alpha_{z z}}{3}
\end{aligned}
$$

$$
\begin{gathered}
\Delta \alpha=2^{-1 / 2}\left[\left(\alpha_{x x}-\alpha_{y y}\right)^{2}+\left(\alpha_{y y}-\alpha_{z z}\right)^{2}+\left(\alpha_{z z}-\alpha_{x x}\right)^{2}+\left(\alpha_{x y}^{2}+\alpha_{y z}^{2}+\alpha_{x z}^{2}\right)\right]^{1 / 2} \\
\beta_{0}=\left(\beta_{x}^{2}+\beta_{y}^{2}+\beta_{z}^{2}\right)^{1 / 2}
\end{gathered}
$$

Many organic molecules, containing conjugated $\pi$ electrons are characterized by large values of molecular first hyper polarizabilities, were analyzed by means of vibrational spectroscopy [43-46]. The intra molecular charge transfer from the donor to acceptor group through a single-double bond conjugated path can induce large variations of both the molecular dipole moment and the molecular polarizability, making IR and Raman activity strong at the same time [47].

Table 3 lists the computed dipole moment $(\mu)$, polarizability $(\alpha)$ and first order hyperpolarizability $\left(\beta_{0}\right)$ as, 1.832 Debye, $0.840 \times 10-30$ esu and $6.477 \times 10^{-30}$ esu, respectively. The first hyperpolarizability $\left(\beta_{0}\right)$ of the title molecule is fifteen times higher than that of urea; hence this molecule has considerable NLO activity. In this molecule, the $\pi-\pi^{*}$ interaction plays a major role in intra-molecular charge transfer and hence the hyperpolarizability of the molecule being increased.

\section{UV-Visible Spectral Studies and HOMO-LUMO Analysis}

The ultraviolet absorption spectrum was obtained in the range of 200-500 $\mathrm{nm}$ to study the electronic properties of Naringin. The UV pattern was taken from a 10-5 molar solution of Naringin dissolved in methanol. TD-DFT calculation was performed to examine the electronic excitations within the MOs 
Table 3: The electric dipole moments $(\mu)$, polarizability $(\boldsymbol{\alpha})$ and hyperpolarizability $\left(\boldsymbol{\beta}_{0}\right)$ values of Naringin.

\begin{tabular}{|c|c|}
\hline Parameters & B3LYP/6-31G(d,p) \\
\hline Dipole moment $(\mu)$ & Debye \\
\hline$\mu_{x}$ & -1.774 \\
\hline$\mu_{y}$ & -0.165 \\
\hline$\mu_{z}$ & 0.423 \\
\hline$\mu$ & 1.832 \\
\hline Polarizability $(\alpha)$ & $\mathbf{x 1 0 ^ { - 3 0 }}$ esu \\
\hline$\alpha_{x x}$ & 396.795 \\
\hline$\alpha_{x y}$ & -40.462 \\
\hline$\alpha_{\mathrm{yy}}$ & 362.008 \\
\hline$\alpha_{x z}$ & 6.148 \\
\hline$\alpha_{y z}$ & 18.176 \\
\hline$\alpha_{z z}$ & 246.065 \\
\hline$\alpha$ & 0.840 \\
\hline Hyperpolarizability $\left(\beta_{0}\right)$ & $\mathrm{x} 10^{-30} \mathrm{esu}$ \\
\hline$\beta_{\mathrm{xxx}}$ & -790.564 \\
\hline$\beta_{x x y}$ & -511.101 \\
\hline$\beta_{\mathrm{xyy}}$ & 210.872 \\
\hline$\beta_{y y y}$ & 49.444 \\
\hline$\beta_{x x z}$ & -84.623 \\
\hline$\beta_{\mathrm{xyz}}$ & 50.617 \\
\hline$\beta_{\mathrm{yyz}}$ & -14.420 \\
\hline$\beta_{\mathrm{xzz}}$ & 4.864 \\
\hline$\beta_{y z z}$ & -8.700 \\
\hline$\beta_{z z z}$ & -2.629 \\
\hline$\beta_{0}$ & 6.477 \\
\hline
\end{tabular}

of Naringin. The both experimental UV and computed results are listed in Table 4. In this work the observed band gaps are about $328.00,282.60,279.80 \mathrm{~nm}$ and computed band gap are about $344.74,293.04,279.63 \mathrm{~nm}$. These values clearly denote that the excitation lay among the conjugative bonds. The Figure 4 shows the absorption spectrum (in methanol) of Naringin. The frontier molecular orbitals are shown in Figure S1 (Supplementary information). The HOMO is located over the 2px orbitals of carbon and oxygen atom in ring D, E and LUMO states appears in ring A. In addition to that the neighboring HOMO-1,-2,-3 and LUMO+1, $+2+3$ are also shown in Figure S1. The estimated HOMO energy is $-5.869 \mathrm{eV}$, and LUMO energy is $-1.034 \mathrm{eV}$ and the HOMO-LUMO energy gap is $-4.835 \mathrm{eV}$.

\section{Summary and Conclusion}

In the present investigation we investigate the structural, spectral and molecular orbitals (MO's) properties of naringin. Naringin consists of naringenin (ring A-C), D-glucose (ring D) and L-rhamnose (ring E). The molecular geometry was studied and found the intra molecular hydrogen bonding between carbonyl and hydroxyl group $\left(\mathrm{C}_{4}=\mathrm{O}_{14} \ldots \mathrm{H}_{16}-\mathrm{O}_{15}\right)$. Vibrational behavior of Naringin was studied by FT-IR and FT-Raman spectra, these spectral values were compared with computed vibrational wavenumbers, agreement is good and discussed the discrepancies among wavenumbers. Furthermore, inter and intra molecular charge transfers were measured by NBO analysis, this analysis clearly showed the maximum energy exchange occurs among $\pi-\pi^{*}$ interactions. The calculated first order hyperpolarizability $\left(6.477 \times 10^{-30} \mathrm{esu}\right)$ is fifteen times higher than that of urea; hence this molecule has a considerable nonlinear optical property. The obtained UV spectrum show excitations at 328.00, 282.60, 279.80 $\mathrm{nm}$, this clearly denotes the excitation lay among the conjugative bonds and calculated HOMO is located over the 2px orbitals of carbon and oxygen atom in ring D, E and LUMO states appears in ring $\mathrm{A}$, whereas, the band gap energy has calculated as $-4.835 \mathrm{eV}$.

Table 4: The electronic transition of Naringin using TD-B3LYP/6-31G(d,p) level.

\begin{tabular}{|c|c|c|c|}
\hline Calculated at B3LYP/ 6-31G(d,p) & Oscillator strength & Experimental Band gap (nm) & Calculated Band gap $(\mathrm{eV} / \mathrm{nm})$ \\
\hline Excited state 1 & Singlet-A/f=0.0010 & 328.00 & $3.5964 \mathrm{eV} / 344.74 \mathrm{~nm}$ \\
\hline 151->154 (HOMO - -LUMO) & & & -5.203 \\
\hline 153->154 (HOMO- LUMO) & & & -4.835 \\
\hline Excited State 2 & Singlet-A /f=0.0499 & 282.60 & $4.2310 \mathrm{eV} / 293.04 \mathrm{~nm}$ \\
\hline $150->154\left(\mathrm{HOMO}_{-3}-\right.$ LUMO $)$ & & & -5.263 \\
\hline $150->156\left(\mathrm{HOMO}_{-3}-\mathrm{LUMO}_{+2}\right)$ & & & -6.153 \\
\hline $150->157\left(\mathrm{HOMO}_{-3}-\mathrm{LUMO}_{+3}\right.$ & & & -5.939 \\
\hline $152->154$ (HOMO $_{-1}$-LUMO & & & -4.917 \\
\hline 153 ->154(HOMO-LUMO & & & -4.835 \\
\hline Excited State 3 & Singlet- $A / f=0.0252$ & 279.80 & $4.4338 \mathrm{eV} / 279.63 \mathrm{~nm}$ \\
\hline 151 ->154(HOMO - - LUMO) & & & -5.203 \\
\hline $152->154\left(\right.$ HOMO $_{-1}$-LUMO) & & & -4.917 \\
\hline 153 ->154(HOMO-LUMO) & & & -4.835 \\
\hline
\end{tabular}




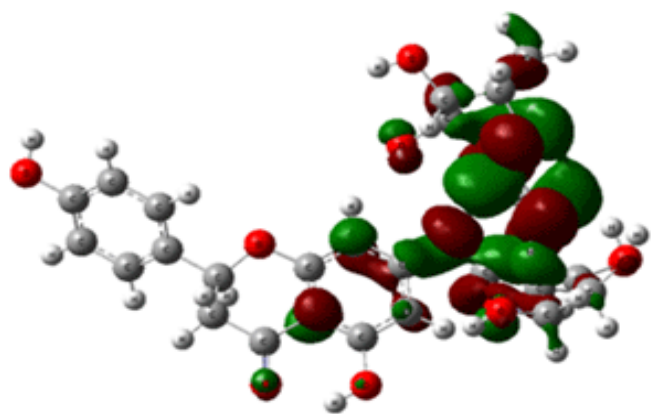

$\mathrm{HOMO}$

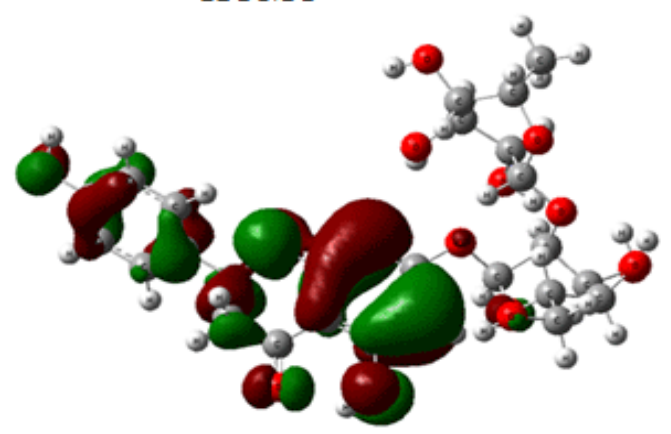

HOMO-1

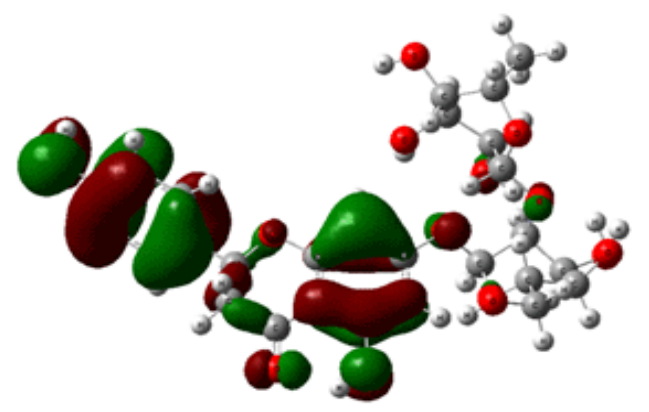

$\mathrm{HOMO}-2$

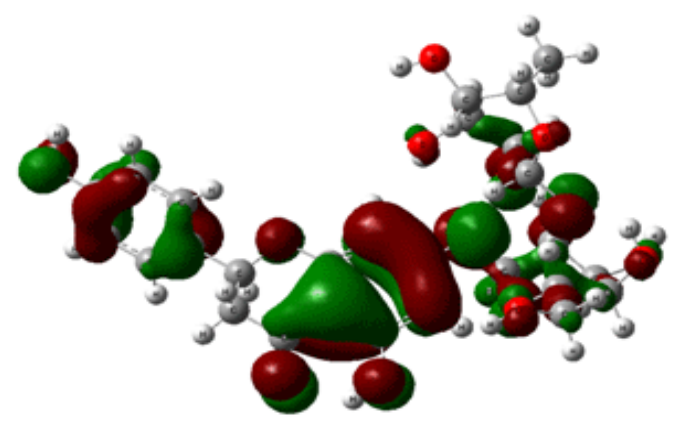

HOMO -3

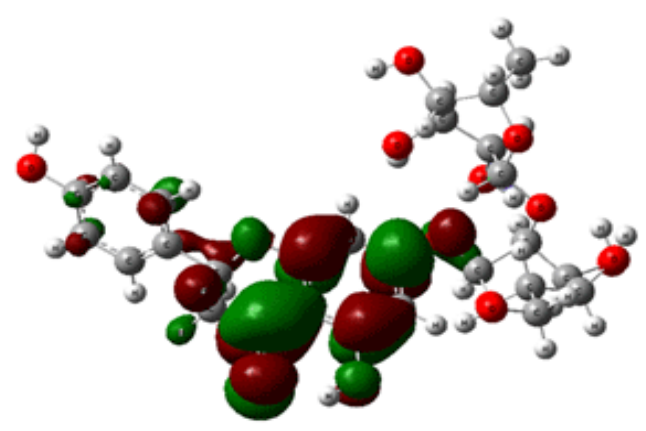

LUMO

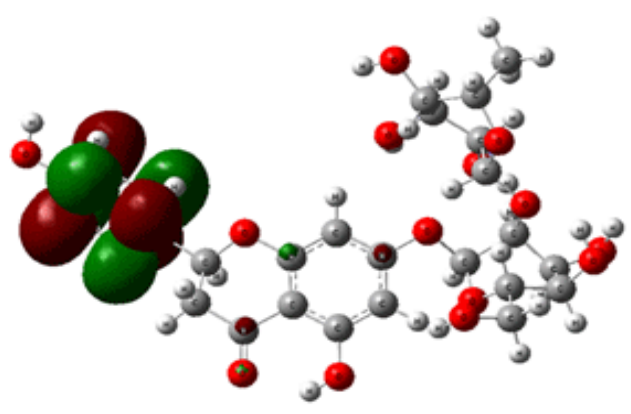

$\mathrm{LUMO}+1$
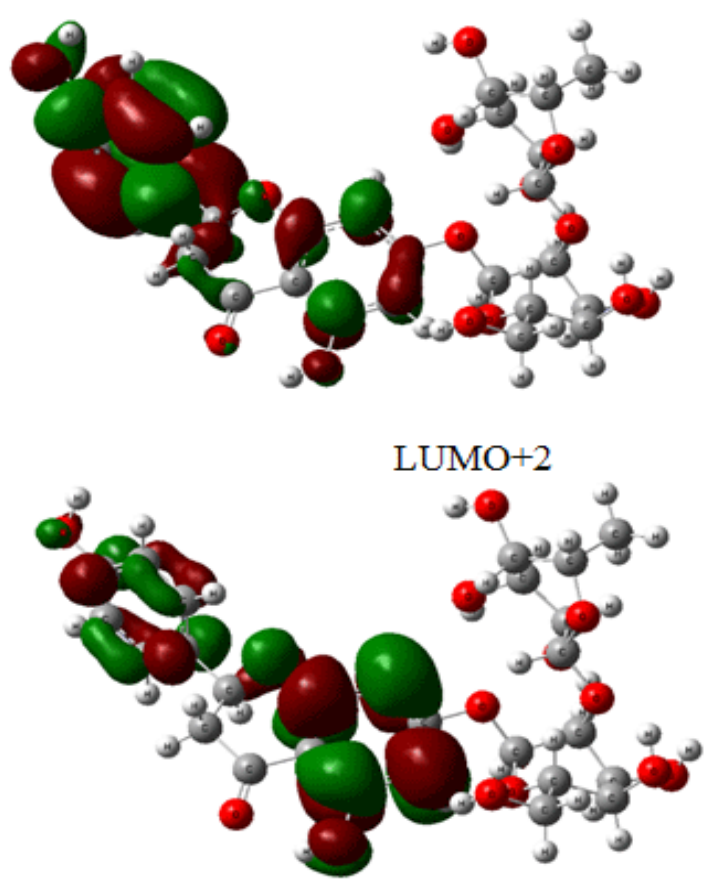

$\mathrm{LUMO}+3$

Figure S1: The Frontier molecular orbitals of Naringin. 


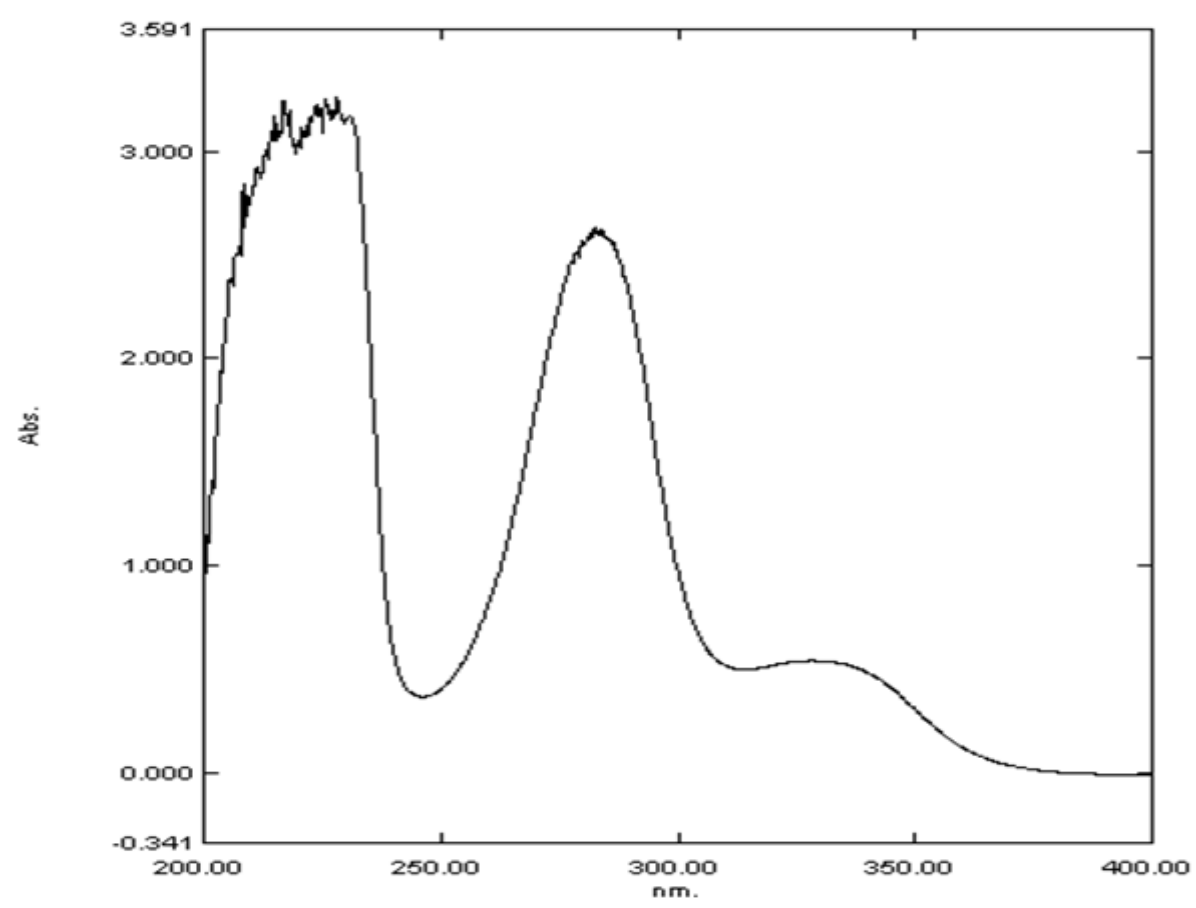

Figure 4: The UV-Visible spectrum is taken from a 10-5 molar solution of Naringin dissolved in methanol.

\section{References}

1. Cook NC and Samman S. Flavonoids-Chemistry, metabolism, cardioprotective effects, and dietary sources. J Nutr Biochem. 1996;7(2):66-76.

2. Ribeiro MH. Naringinases: occurrence, characteristics, and applications. Appl Microbiol Biotechnol. 2011;90(6):1883-1895. Doi: 10.1007/ s00253-011-3176-8.

3. Renugadevi J and Prabu SM. Naringenin protects against cadmiuminduced oxidative renal dysfunction in rats. Toxicology. 2009;256(12):128-134.

4. Choudhury R, Chowrimootoo G, Srai K and Debnam E, Rice-Evans CA. Interactions of the Flavonoid Naringenin in the Gastrointestinal Tract and the Influence of Glycosylation. Biochem Biophys Res Commun. 1999;265(2):410-415.

5. Parini M, Braquet P and Garany RP. Heterogenous effect of flavonoids on $\mathrm{K}+$ loss and lipid peroxidation induced by oxygen-free radicals in human red cells. Pharmacol. Res. Commun. 1986;18(1) 61-72.

6. Jung G. Hennings G, Pfeifer M, and BesslerWG. Iteraction of metalcomplexing coumpounds with lymphocytes and lympho cell lines. Mol. Pharmacol. 1983;23(3):698-702.

7. Jagetia GC, Venkatesha VA and Reddy TK. Naringin, a citrus flavonone, protects against radiation-induced chromosome damage in mouse bone marrow. Mutagenesis. 2003;13(4):337-343.

8. Schindler $\mathrm{R}$ and Mentlein R. Flavonoids and vitamin E reduce the release of the angiogenic peptide vascular endothelial growth factor from human tumor cells. J. Nutr. 2006;136(6):1477-1482.

9. Martin MJ, Marhuenda E, Perez-Guerrero C and Franco JM. Antiulcer Effect of Naringin on Gastric Lesions Induced by Ethanol in Rats. Pharmacology. 1994;49(3):144-150.
10. Gaussian 03 program, (Gaussian Inc., Walling ford CT) 2004.

11.Schlegel HB. Optimization of equilibrium geometries and transition structures J. Comput. Chem. 1982;3(2):214-218.

12.Frisch NAB and Holder AJ. GAUSSVIEW User Manual, Gaussian Inc, Pittsburgh PA. 2000.

13.Rauhut G and Pulay G. Transferable Scaling Factors for Density Functional Derived Vibrational Force Fields. J. Phys. Chem. 1995;99(10):3093-3100.

14.Michalska D. Raint Program. Wroclaw University of Technology.2003.

15.Michalska D and Wysokinski R. The prediction of Raman spectra of platinum(II) anticancer drugs by density functional theory. Chem. Phys. Lett. 2005;403(1-3):211-217.

16.Wera M, Serdiuk LE, Roshal AD and Jerzy B. ejowski . 3-Hydroxy2-(4-methoxyphenyl)-4Hchromen-4-one J. Blazejowski. Acta Cryst. 2011;E67(o440).

17.Zhang ZT and Zhang XL. F-type or T-type Aromatic-Aromatic Interaction in Two Isoflavone Derivatives. J. Chem. Crystallogr. 2008;38(2):129-133.

18.Silverstein M, Basseler CG and Morill CT. Spectrometric Identification of Organic Compounds. Wiley: New York, 1981.

19.Krishnakumar V, Murugeswari K, Prabavathi N and Mathammal R. Molecular structure, vibrational spectra, HOMO, LUMO and NMR studies of 2-chloro-4-nitrotoluene and 4-chloro-2-nitrotoluene. Spectrochim. Acta A. 2012;91:1-10.

20.Varsanyi G. Vibrational Spectra of Benzene Derivatives. Academic Press: New York. 1969.

21.Colthup NB, Daly LH and Wiberly SE. Introduction to Infrared and Raman spectroscopy. Academic Press: New York. 1990. 
22.Erdoğdu Y and Güllüoglu MT. Analysis of vibrational spectra of 2 and 3-methylpiperidine based on density functional theory calculations. Spectrochim. Acta. 2009;74(1):162-167.

23.Roeges NPG. A Guide to the Complete Interpretation of Infrared Spectra of Organic Structures. Wiley: New York.1994.

24.Varghese HT, Paniker CY, Madhavan VS, Mathew S, Vinsova J and Alsenoy CV. FT-IR, FT-Raman and DFT calculations of the salicylanilide derivate 4-chloro-2-(4-bromophenylcarbamoyl)phenyl acetate. J. Raman. Spectrosc. 2009;40(9):1211-1223.

25.Sundaraganesan N, Meganathan C, Saleem H and Dominic Joshua B. Vibrational spectroscopy investigation using ab initio and density functional theory analysis on the structure of 5-amino-o-cresol. Spectrochim. Acta A. 2007;68(3):619-625.

26.Sebastien $S$ and Sundaraganesan N. The spectroscopic (FT-IR, FT-IR gas phase, FT-Raman and UV) and NBO analysis of 4-Hydroxypiperidine by density functional method. Spectrochim. Acta A. 2010;75(3):941-952.

27.Ramalingam M, Joccob M, Venuvanlingam P and Sundaraganesan N. Harmonic analysis of vibrations of morpholine-4-ylmethylthiourea: A DFT, midinfrared and Raman spectral study. Spectrochim. Acta A 2008;71(3):996-1002.

28.Pavia DL, Lampman GM and Kriz GS. Introduction to Spectroscopy. Third Edition, Thompson Learning, US. 2001.

29. Oliveira RN, Mancini MC, Oliveira FCS, Passos TM, Quility B and Thiré B, et. al. FTIR analysis and quantification of phenols and flavonoids of five commercially available plants extracts used in wound healing Revista Matéria. 2016;21(3):767-779.

30.Akkaya $Y$ and Akyüz S. Infrared and Raman spectra, ab initio calculations vibrational assignment of 4-aminosalicylic acid. Vib. Spectrosc. 2006;42(2):292-301.

31.Nogueira HIS. Surface-enhanced Raman scattering (SERS) of 3-aminosalicylic and 2-mercaptonicotinic acids in silver colloids. Spectrochim. Acta A. 1998;54(10):1461-1470.

32.Sundaraganesan N, Meganathan C, Anand B and Lapouge C. FTIR, FT-Raman spectra and ab initio DFT vibrational analysis of p-bromophenoxyacetic acid. Spectrochim. Acta A. 2007;66(3):773-780.

33.Erdoğdu Y, Unsalan $O$ and Güllüoglu MT. FT-Raman, FT-IR spectral and DFT studies on 6, 8-dichloroflavone and 6, 8-dibromoflavone. J. Raman Spectrosc. 2010;41(7):820-828.

34.Puri M, Kurt A, Schwarz WH, Singh S and Kennedy JF. Molecular characterization and enzymatic hydrolysis of naringin extracted from kinnow peel waste. Int. J. Bio. Macromol. 2011;48(1):58-62.
35.Subramanian MK, Anbarasan PM and Manimegali S. Molecular structure, NMR and vibrational spectral analysis of 2,4-difluorophenol by ab initio HF and density functional theory. J. Raman Spectrosc. 2009;40(11):1657-1663.

36.Ficarra R, Tommasini S, Raneri D, Calabrò ML, Di Bella MR and Rustichelli C, et. al. Study of flavonoids/beta-cyclodextrins inclusion complexes by NMR, FT-IR, DSC, X-ray investigation. J Pharm Biomed Anal. 2002;29(6):1005-1014

37.Reed AE, and Weinhold F. Natural bond orbital analysis of nearHartree-Fock water dimer. J. Chem. Phys. 1983;78(6):4066-4073.

38.Reed AE, and Weinhold F. Natural localized molecular orbitals. J. Chem. Phys. 1985;83(4):1736-1740.

39.Reed AE, Weinstock RB and Weinhold F. Natural population analysis. J.Chem. Phys. 1985;83(2):735-746.

40.Foster JP and Wienhold F. Natural hybrid orbitals. J. Am. Chem. Soc. 1980;102(24):7211-7218.

41.Chocholousova J. Vladimir Spirko J and Hobza P. First local minimum of the formic acid dimer exhibits simultaneously red-shifted $\mathrm{O}-\mathrm{H} \cdots \mathrm{O}$ and improper blue-shifted C-H $\cdots 0$ hydrogen bonds. Phys. Chem. Chem. Phys. 2004;6(1):37-41.

42.Colthup NB, Daly LH and Wiberly SE, Introduction to Infrared and Raman Spectroscopy. Academic Press: New York, 1990.

43.Castiglioni C, Del zoppo M, Zuliani P and Zerbi G. Experimental molecular hyperpolarizabilities from vibrational spectra in systems with large electron-phonon coupling. Synth. Met. 1995;74(2):171-177.

44.Del zoppo M, Castiglioni C and Zerbi G, Non-Linear Opt. 1995;9:73.

45.Del zoppo M, Castiglioni C, Zuliani P, Razelli A, Zerbi G and BlanchardDesce M. Use of vibrational spectra for the determination of first-order molecular hyperpolarizabilities of push-pull polyenes as function of structural parameters. J. Appl. Polym. Sci. 1998;70 (7):1311-1320.

46.Zuliani P, Del zoppo M, Castiglioni C and Zerbi G. Solvent effects on first-order molecular hyperpolarizability: A study based on vibrational observables. Chem. Phys. 1995;103(23):9935.

47.Ravikumar C, Huber Joe I and Jayakumar VS, Charge transfer interactions and nonlinear optical properties of push-pull chromophore benzaldehyde phenylhydrazone: A vibrational approach. Chem. Phys. Lett. 2008;460(4-6):552-558. 